\title{
Post-processing procedures for an elliptic distributed optimal control problem with pointwise state constraints ${ }^{\vec{n}}$
}

\author{
Susanne C. Brenner ${ }^{\mathrm{a}, *}$, Li-Yeng Sung ${ }^{\mathrm{a}}$, Yi Zhang ${ }^{\mathrm{b}}$ \\ ${ }^{a}$ Department of Mathematics and Center for Computation and Technology, Louisiana State University, Baton Rouge, LA 70803 \\ ${ }^{b}$ Department of Mathematics, University of Tennessee, Knoxville, TN 37996
}

\begin{abstract}
We consider an elliptic distributed optimal control problem with state constraints and compare three post-processing procedures that compute approximations of the optimal control from the approximation of the optimal state obtained by a quadratic $C^{0}$ interior penalty method.
\end{abstract}

Keywords: elliptic optimal control, state constraints, quadratic $C^{0}$ interior penalty method, fourth order variational inequality, post-processing

2008 MSC: $65 \mathrm{~K} 15,65 \mathrm{~N} 30$

\section{Introduction}

Let $\Omega \subseteq \mathbb{R}^{2}$ be a bounded convex polygonal domain, $y_{d} \in L_{2}(\Omega)$ and $\beta$ be a positive number. We consider a model elliptic distributed optimal control problem with pointwise state constraints (cf. [15]):

$$
\begin{aligned}
\text { minimize } & J(y, u)=\frac{1}{2} \int_{\Omega}\left(y-y_{d}\right)^{2} d x+\frac{\beta}{2} \int_{\Omega} u^{2} d x \\
\text { over } & (y, u) \in H_{0}^{1}(\Omega) \times L_{2}(\Omega) \\
\text { subject to } & \left\{\begin{aligned}
-\Delta y=u & \text { in } \Omega \\
y \leq \psi & \text { a.e. in } \Omega
\end{aligned}\right.
\end{aligned}
$$

We assume that $\psi \in C^{2}(\bar{\Omega})$ and $\psi>0$ on $\partial \Omega$. Here and below we will follow standard notation for differential operators and Sobolev spaces that can be found for example in [17,9].

Since $\Omega$ is convex, it follows from the elliptic regularity theory [25, 18, 35] that the state $y$ belongs to $H^{2}(\Omega) \cap$ $H_{0}^{1}(\Omega)$ and hence, by replacing the control $u$ with $-\Delta y$ in (1.1), we can instead look for the minimizer of the reduced functional

$$
G(y)=\frac{1}{2} \int_{\Omega}\left(y-y_{d}\right)^{2} d x+\frac{\beta}{2} \int_{\Omega}(\Delta y)^{2} d x
$$

in the closed convex set $K=\left\{y \in H^{2}(\Omega) \cap H_{0}^{1}(\Omega): y \leq \psi\right.$ in $\left.\Omega\right\}$.

Note that [26, Theorem 2.2.1]

$$
\int_{\Omega}(\Delta v)(\Delta w) d x=\int_{\Omega}\left(D^{2} v: D^{2} w\right) d x \quad \forall v, w \in H^{2}(\Omega) \cap H_{0}^{1}(\Omega),
$$

\footnotetext{
This work was supported in part by the National Science Foundation under Grant Nos. DMS-10-16332 and DMS-13-19172.

* corresponding author

Email addresses: brenner@math.1su.edu (Susanne C. Brenner), sung@math.lsu.edu (Li-Yeng Sung), yzhan112@utk.edu (Yi Zhang)
} 
where $D^{2} v: D^{2} w=\sum_{i, j=1}^{2} v_{x_{i} x_{j}} w_{x_{i} x_{j}}$ is the inner product of the Hessian matrices of $v$ and $w$. Therefore, after a simple manipulation, we have the following equivalent minimization problem:

$$
\text { Find } \quad \bar{y}=\underset{y \in K}{\operatorname{argmin}}\left[\frac{1}{2} \mathcal{A}(y, y)-\left(y_{d}, y\right)\right],
$$

where $(\cdot, \cdot)$ is the inner product of $L_{2}(\Omega)$ and

$$
\mathcal{A}(v, w)=\int_{\Omega}\left[\beta\left(D^{2} v: D^{2} w\right)+v w\right] d x .
$$

Since the symmetric bilinear form $\mathcal{A}(\cdot, \cdot)$ is coercive on $H^{2}(\Omega) \cap H_{0}^{1}(\Omega)$ by a Poincaré-Friderichs inequality [37] and $K$ is a closed convex subset of $H^{2}(\Omega) \cap H_{0}^{1}(\Omega)$ by the Sobolev inequality [1], it follows from the standard theory $[32,29,38,23]$ that $(1.2)$ has a unique solution characterized by the fourth order variational inequality

$$
\mathcal{A}(\bar{y}, y-\bar{y}) \geq\left(y_{d}, y-\bar{y}\right) \quad \forall y \in K .
$$

According to the regularity results in $[21,22,14]$, the solution $\bar{y}$ of (1.2) belongs to $C^{2}(\Omega) \cap H_{\text {loc }}^{3}(\Omega)$. On the other hand, since the state constraint is inactive near $\partial \Omega$ because $\psi>0$ on $\partial \Omega$, the variational inequality (1.4) becomes an equality near $\partial \Omega$. Therefore the regularity of $\bar{y}$ near $\partial \Omega$ is determined by the elliptic regularity of the biharmonic equation with the boundary conditions of simply supported plates (cf. [4] and [13, Appendix A]). Hence $\bar{y}$ belongs to $H^{2+\alpha}$ in a neighborhood of $\partial \Omega$, where the elliptic regularity index $\alpha \in(0,2]$ is determined by the interior angles of $\Omega$. However $\bar{u}=-\Delta \bar{y}$ belongs to $H_{0}^{1}(\Omega)$ since the singularities of $\bar{y}$ at the corners of $\Omega$ are harmonic functions.

We can rewrite the variational inequality (1.4) in an equivalent form by using a Lagrange multiplier:

$$
\begin{aligned}
& \mathcal{A}(\bar{y}, y)=\left(y_{d}, y\right)-\int_{\Omega} y d \bar{\lambda} \quad \forall y \in H^{2}(\Omega) \cap H_{0}^{1}(\Omega), \\
& \int_{\Omega}(\psi-\bar{y}) d \bar{\lambda}=0,
\end{aligned}
$$

where $\bar{\lambda}$ is a nonnegative finite Borel measure.

The fact that the Lagrange multiplier $\bar{\lambda}$ is a measure and not a function in $L_{2}(\Omega)$ complicates the analysis of finite element methods for $(1.2) /(1.4)$ considerably. Following the ideas introduced in $[12,11]$ for the obstacle problem of clamped Kirchhoff plates, a quadratic $C^{0}$ interior penalty method for $(1.2) /(1.4)$ was investigated in [13], where it was shown, without using (1.5), that the error for the approximation of the optimal state $\bar{y}$ in an $H^{2}$-like energy norm is $O\left(h^{\alpha}\right)$ on quasi-uniform meshes and $O(h)$ on properly graded meshes, where $h$ represents the mesh size.

In this paper we will compare three post-processing procedures that compute approximations of the optimal control $\bar{u}=-\Delta \bar{y}$ from the approximation of $\bar{y}$ obtained by the quadratic $C^{0}$ interior penalty method. The first procedure is based on numerical differentiation, the second procedure involves numerical differentiation and averaging, and the third procedure involves numerical differentiation and smoothing. We will demonstrate that even though the a priori $L_{2}$ error estimates for the approximate optimal controls generated by all three procedures are of the same magnitude as the error for the optimal state in the energy norm, in practice the second procedure performs better than the first one and the third procedure performs better than the second one. In particular the convergence of the approximate optimal control in the $H^{1}$ norm is observed for the third procedure even on domains with singular corners.

The rest of the paper is organized as follows. In Section 2 we review the quadratic $C^{0}$ interior penalty method and introduce the post-processing procedures. Since two of the procedures have been analyzed previously, we will focus on the analysis of the third procedure, which is carried out in Section 3. Numerical results that compare the performance of the post-processing procedures are reported in Section 4. We end the paper with some concluding remarks in Section 5.

Throughout the paper we will use $C$ to denote a generic positive constant independent of the mesh size $h$ that can take different values at different occurrences.

Remark 1.1. The reformulation of optimal control problems as fourth order variational inequalities is well-known in the literature (cf. for example $[3,33,24,36])$. The novelty of our approach is in the analysis, which shows that 
essentially all finite element methods that work for the boundary value problems of Kirchhoff plates also work for the variational inequalities, without assuming additional conditions on the free boundary. It would be interesting to find out whether our approach can be extended to optimal control problems with both state and control constraints that have been investigated in $[34,28,16,30,39]$ by classical approaches.

\section{Post-Processing Procedures for a Quadratic $C^{0}$ Interior Penalty Method}

In this section we recall the quadratic $C^{0}$ interior penalty method in [13] and introduce the post-processing procedures that compute approximations of the optimal control.

\subsection{Triangulations}

We consider two types of simplicial triangulation $\mathcal{T}_{h}$ of $\Omega$ with mesh size $h$. For a quasi-uniform triangulation $\mathcal{T}_{h}$, we have $h_{T} \approx h$ for all $T \in \mathcal{T}_{h}$. Let $p_{1}, \ldots, p_{L}$ be the corners of $\Omega$. For a graded triangulation $\mathcal{T}_{h}$, we have

$$
h_{T} \approx h \Phi\left(c_{T}\right) \quad \forall T \in \mathcal{T}_{h},
$$

where $c_{T}$ is the center of $T$ and

$$
\Phi(x)=\prod_{\ell=1}^{L}\left|p_{\ell}-x\right|^{1-\alpha_{\ell}} .
$$

Here the grading parameters $\alpha_{\ell}>0$ are determined by

$$
\left\{\begin{array}{ll}
\alpha_{\ell}=1 & \text { if } \omega_{\ell} \leq \frac{\pi}{2} \\
\alpha_{\ell}<\left(\frac{\pi}{\omega_{\ell}}\right)-1 & \text { if } \frac{\pi}{2}<\omega_{\ell}<\pi
\end{array},\right.
$$

where $\omega_{\ell}$ is the interior angle at the corner $p_{\ell}$ of $\Omega$.

Remark 2.1. The construction of triangulations that satisfy both condition (2.1) and the minimum angle condition is discussed for example in $[2,7,5]$.

\subsection{Averages and Jumps}

Let $V_{h} \subseteq H_{0}^{1}(\Omega)$ be the quadratic Lagrange finite element space associated with $\mathcal{T}_{h}, \mathcal{E}_{h}$ be the set of the edges of the triangles in $\mathcal{T}_{h}, \mathcal{E}_{h}^{i} \subset \mathcal{E}_{h}$ be the set of the edges interior to $\Omega$, and $\mathcal{E}_{h}^{b}=\mathcal{E}_{h} \backslash \mathcal{E}_{h}^{i}$ be the set of the edges on $\partial \Omega$.

Let $e \in \mathcal{E}_{h}^{i}$ be the common edge of $T_{ \pm} \in \mathcal{T}_{h}$ and $n_{e}$ be the unit normal of $e$ pointing from $T_{-}$to $T_{+}$. For $v \in V_{h}$, we define on $e$

$$
\left\{\left\{\frac{\partial^{2} v}{\partial n^{2}}\right\}\right\}=\frac{1}{2}\left(\left.\frac{\partial^{2} v_{+}}{\partial n_{e}^{2}}\right|_{e}+\left.\frac{\partial^{2} v_{-}}{\partial n_{e}^{2}}\right|_{e}\right) \text { and } \llbracket\left|\frac{\partial v}{\partial n} \rrbracket=\frac{\partial v_{+}}{\partial n_{e}}\right|_{e}-\left.\frac{\partial v_{-}}{\partial n_{e}}\right|_{e},
$$

where $v_{ \pm}$is the restriction of $v$ to $T_{ \pm}$. These definitions are independent of the choices of $T_{ \pm}$.

Similarly we define on $e$

$$
\left\{\left\{\frac{\partial v}{\partial n_{e}}\right\}\right\}=\frac{1}{2}\left(\left.\frac{\partial v_{+}}{\partial n_{e}}\right|_{e}+\left.\frac{\partial v_{-}}{\partial n_{e}}\right|_{e}\right), \quad \llbracket\left|\frac{\partial^{2} v}{\partial n_{e}^{2}} \rrbracket=\frac{\partial^{2} v_{+}}{\partial n_{e}^{2}}\right|_{e}-\left.\frac{\partial^{2} v_{-}}{\partial n_{e}^{2}}\right|_{e}, \quad \llbracket\left|\frac{\partial^{2} v}{\partial n_{e} \partial t_{e}} \rrbracket=\frac{\partial^{2} v_{+}}{\partial n_{e} \partial t_{e}}\right|_{e}-\left.\frac{\partial^{2} v_{-}}{\partial n_{e} \partial t_{e}}\right|_{e},
$$

where $t_{e}$ is the unit tangent of $e$ obtained by rotating $n_{e}$ by a counterclockwise right angle. These definitions, which only appear in the analysis, depend on the choices of $T_{ \pm}$.

For a boundary edge $e \in \mathcal{E}_{h}^{b}$, we take $n_{e}$ to be the unit normal pointing towards the outside of $\Omega$ and define on $e$

$$
\left\{\left\{\frac{\partial v}{\partial n_{e}}\right\}\right\}=\left.\frac{\partial v}{\partial n_{e}}\right|_{e} \text { and } \llbracket\left|\frac{\partial^{2} v}{\partial n_{e}^{2}} \rrbracket=-\frac{\partial^{2} v}{\partial n_{e}^{2}}\right|_{e} .
$$

We note that $\llbracket \partial v / \partial n \rrbracket$ on an edge in $\mathcal{E}_{h}^{i}$ and $\left.\left\{\partial \partial v / \partial n_{e}\right\}\right\}$ on an edge in $\mathcal{E}_{h}$ are well defined for any function $v \in$ $H^{2}\left(\Omega ; \mathcal{T}_{h}\right)$, where

$$
H^{2}\left(\Omega ; \mathcal{T}_{h}\right)=\left\{v \in L_{2}(\Omega):\left.v\right|_{T} \in H^{2}(T) \quad \forall T \in \mathcal{T}_{h}\right\}
$$

is the space of piecewise $H^{2}$ functions. 


\subsection{Discrete Problem}

$C^{0}$ interior penalty methods are discontinuous Galerkin methods originally designed for fourth order elliptic boundary value problems $[20,10]$. The symmetric bilinear form $a_{h}(\cdot, \cdot)$ for $C^{0}$ interior penalty methods for simply supported plates [8] is given by

$$
\begin{aligned}
a_{h}(w, v)= & \sum_{T \in \mathcal{T}_{h}} \int_{T}\left(D^{2} w: D^{2} v\right) d x+\sum_{e \in \mathcal{E}_{h}^{i}} \int_{e}\left\{\left\{\partial^{2} w / \partial n^{2}\right\} \llbracket \partial v / \partial n \rrbracket d s\right. \\
& +\sum_{e \in \mathcal{E}_{h}^{i}} \int_{e}\left\{\left\{\partial^{2} v / \partial n^{2}\right\}\right\} \llbracket \partial w / \partial n \rrbracket d s+\sigma \sum_{e \in \mathcal{E}_{h}^{i}}|e|^{-1} \int_{e} \llbracket \partial w / \partial n \rrbracket \llbracket \partial v / \partial n \rrbracket d s \quad \forall w, v \in V_{h},
\end{aligned}
$$

where $|e|$ is the length of $e$ and $\sigma$ is a positive penalty parameter. We assume $\sigma$ is sufficiently large so that $a_{h}(\cdot, \cdot)$ is positive definite on $V_{h}$. (The choice of $\sigma$ depends on the shape regularity of the triangulation.)

Remark 2.2. Let $\phi \in H^{-1}(\Omega)=\left[H_{0}^{1}(\Omega)\right]^{\prime}$. The variational problem

$$
a(z, v)=\phi(v) \quad \forall v \in H^{2}(\Omega) \cap H_{0}^{1}(\Omega),
$$

where

has a consistent and stable discretization given by

$$
a(z, v)=\int_{\Omega}(\Delta z)(\Delta v) d x=\int_{\Omega}\left(D^{2} z: D^{2} v\right) d x,
$$

$$
a_{h}\left(z_{h}, v\right)=\phi(v) \quad \forall v \in V_{h} .
$$

The bilinear form $\mathcal{A}_{h}(\cdot, \cdot)$ on $V_{h}$ is then defined by

$$
\mathcal{A}_{h}(w, v)=\beta a_{h}(w, v)+(w, v) .
$$

Let $\mathcal{V}_{h}$ be the set of the vertices of the triangles in $\mathcal{T}_{h}$ and $K_{h}=\left\{v \in V_{h}: v(p) \leq \psi(p)\right.$ for all $\left.p \in \mathcal{V}_{h}\right\}$. The discrete problem for (1.2) is:

$$
\text { Find } \quad \bar{y}_{h}=\underset{y_{h} \in K_{h}}{\operatorname{argmin}}\left[\frac{1}{2} \mathcal{A}_{h}\left(y_{h}, y_{h}\right)-\left(y_{d}, y_{h}\right)\right] \text {. }
$$

Since $\mathcal{A}_{h}(\cdot, \cdot)$ is symmetric positive definite, the discrete problem (2.6) has a unique solution characterized by

$$
\mathcal{A}_{h}\left(\bar{y}_{h}, y_{h}-\bar{y}_{h}\right) \geq\left(y_{d}, y_{h}-\bar{y}_{h}\right) \quad \forall y_{h} \in K_{h} .
$$

\subsection{Error Estimates}

Let the mesh-dependent norm $\|\cdot\|_{h}$ on $V_{h}$ be defined by

$$
\|v\|_{h}^{2}=\beta\left(\sum_{T \in \mathcal{T}_{h}}|v|_{H^{2}(T)}^{2}+\sum_{e \in \mathcal{E}_{h}^{i}}|e|^{-1}\|\llbracket \partial v / \partial n \rrbracket\|_{L_{2}(e)}^{2}\right)+\|v\|_{L_{2}(\Omega)}^{2} .
$$

Since we assume $\sigma$ is sufficiently large, the norm $\|\cdot\|_{h}$ is equivalent to the norm $\mathcal{A}_{h}(\cdot, \cdot)^{\frac{1}{2}}$ induced by the bilinear form $\mathcal{A}_{h}$.

The main results in [13] are summarized in the following theorem (cf. Theorem 5.1, Corollary 5.1 and Theorem 5.2 in [13]).

Theorem 2.3. There exists a positive constant $C$ independent of $h$ such that

$$
\begin{aligned}
\left\|\bar{y}-\bar{y}_{h}\right\|_{h} & \leq C h^{\tau}, \\
\left|\bar{y}-\bar{y}_{h}\right|_{H^{1}(\Omega)}+\left\|\bar{y}-\bar{y}_{h}\right\|_{L_{\infty}(\Omega)} & \leq C h^{\tau},
\end{aligned}
$$

where $\tau=\alpha$ if $\mathcal{T}_{h}$ is quasi-uniform and $\tau=1$ if $\mathcal{T}_{h}$ is graded according to (2.1)-(2.3).

Remark 2.4. Since the norms $|\cdot|_{H^{1}(\Omega)}$ and $\|\cdot\|_{L_{\infty}(\Omega)}$ are weaker than the $H^{2}$-like norm $\|\cdot\|_{h}$, the estimate (2.10) is not sharp, which is confirmed by numerical experiments (cf. [13]). 


\subsection{Post-Processing Procedures}

We can compute approximations of the optimal control $\bar{u}=-\Delta \bar{y}$ by post-processing the approximation $\bar{y}_{h}$ of the optimal state $\bar{y}$ defined in (2.6). From here on the constant $\tau$ equals $\alpha$ if $\mathcal{T}_{h}$ is quasi-uniform and $\tau$ equals 1 if $\mathcal{T}_{h}$ is graded according to (2.1)-(2.3).

\section{First Procedure}

We take $\bar{u}_{h, 1}=-\Delta_{h} \bar{y}_{h}$ to be the piecewise constant function where $\Delta_{h}$ is the piecewise Laplacian operator. This is the most straightforward procedure and it follows immediately from (2.8) and (2.9) that

$$
\left\|\bar{u}-\bar{u}_{h, 1}\right\|_{L_{2}(\Omega)} \leq C h^{\tau} .
$$

\section{Second Procedure}

Observe that

$$
(\nabla \bar{y}, \nabla v)=(\bar{u}, v) \quad \forall v \in H_{0}^{1}(\Omega)
$$

because $\bar{u}=-\Delta \bar{y}$. Therefore we define $\bar{u}_{h, 2} \in V_{h}$ by the equation

$$
\left(\nabla \bar{y}_{h}, \nabla v\right)=\left(\bar{u}_{h, 2}, v\right) \quad \forall v \in V_{h} .
$$

This procedure was analyzed in [13]. We have ([13, Theorem 5.3])

$$
\left\|\bar{u}-\bar{u}_{h, 2}\right\|_{L_{2}(\Omega)} \leq C h^{\tau} .
$$

\section{Third Procedure}

Since $\bar{u}=-\Delta \bar{y} \in H_{0}^{1}(\Omega)$, it follows from integration by parts that

$$
(\nabla \bar{u}, \nabla v)=(\Delta \bar{y}, \Delta v)=\int_{\Omega}\left(D^{2} \bar{y}: D^{2} v\right) d x \quad \forall v \in H^{2}(\Omega) \cap H_{0}^{1}(\Omega) .
$$

Motivated by Remark 2.2 and (2.14), we define $\bar{u}_{h, 3} \in V_{h}$ by the equation

$$
\left(\nabla \bar{u}_{h, 3}, \nabla v\right)=a_{h}\left(\bar{y}_{h}, v\right) \quad \forall v \in V_{h} .
$$

The analysis of this post-processing procedure will be carried out in Section 3. Here we note that there is an alternative definition of $\bar{u}_{h, 3}$. We begin by rewriting (2.7) in an equivalent form (analog of (1.5)) in terms of a Lagrange multiplier:

$$
\begin{aligned}
& \mathcal{A}_{h}\left(\bar{y}_{h}, v\right)=\left(y_{d}, v\right)-\sum_{p \in \mathcal{V}_{h}} c_{h, p} v(p) \quad \forall v \in V_{h}, \\
& \sum_{p \in \mathcal{V}_{h}} c_{h, p}\left(\psi(p)-\bar{y}_{h}(p)\right)=0
\end{aligned}
$$

where $c_{h, p} \geq 0$ for all $p \in \mathcal{V}_{h}$. Let $\bar{\lambda}_{h}$ be the nonnegative measure defined by

$$
\bar{\lambda}_{h}=\sum_{p \in \mathcal{V}_{h}} c_{h, p} \delta_{p},
$$

where $\delta_{p}$ is the Dirac point measure associated with the vertex $p$. Then it follows from (2.5), (2.16a) and (2.17) that

$$
\int_{\Omega} v d \bar{\lambda}_{h}=\left(y_{d}, v\right)-\mathcal{A}_{h}\left(\bar{y}_{h}, v\right)=\left(y_{d}-\bar{y}_{h}, v\right)-\beta a_{h}\left(\bar{y}_{h}, v\right) \quad \forall v \in C(\bar{\Omega}),
$$

which together with (2.15) implies

$$
\left(\nabla \bar{u}_{h, 3}, \nabla v\right)=\frac{1}{\beta}\left[\left(y_{d}-\bar{y}_{h}, v\right)-\int_{\Omega} v d \bar{\lambda}_{h}\right] \quad \forall v \in V_{h} .
$$




\section{Analysis of the Third Post-Processing Procedure}

In this section we will show that $\bar{u}_{h, 3}$ satisfies similar $L_{2}$ error estimates as $\bar{u}_{h, 1}$ and $\bar{u}_{h, 2}$. Throughout this section $\bar{u}_{h, 3}$ will be denoted simply by $\bar{u}_{h}$.

\subsection{Integration by Parts and Interpolation}

We have an integration by parts formula that holds for $v \in V_{h}$ and $w \in H^{2}\left(\Omega ; \mathcal{T}_{h}\right) \cap H_{0}^{1}(\Omega)$ :

$$
\sum_{T \in \mathcal{T}_{h}} \int_{T}\left(D^{2} v: D^{2} w\right) d x=-\sum_{e \in \mathcal{E}_{h}^{i}} \int_{e}\left(\int\left\{\frac{\partial^{2} v}{\partial n^{2}}\right\}\right) \llbracket \llbracket \frac{\partial w}{\partial n} \rrbracket+\llbracket\left[\frac{\partial^{2} v}{\partial t_{e} \partial n_{e}} \rrbracket \frac{\partial w}{\partial t_{e}}\right) d s-\sum_{e \in \mathcal{E}_{h}} \int_{e} \llbracket\left[\frac{\partial^{2} v}{\partial n_{e}^{2}} \rrbracket \int\left\{\frac{\partial w}{\partial n_{e}}\right\}\right\} d s .
$$

Let $\Pi_{h}: H^{2}(\Omega) \cap H_{0}^{1}(\Omega) \longrightarrow V_{h}$ be the nodal interpolation operator for the quadratic Lagrange finite element space. The following interpolation error estimate is well-known [17, 9]:

$$
\sum_{m=0}^{2} h_{T}^{m}\left|\phi-\Pi_{h} \phi\right|_{H^{m}(T)} \leq C h_{T}^{2}|\phi|_{H^{2}(T)} \quad \forall T \in \mathcal{T}_{h}, \phi \in H^{2}(\Omega) \cap H_{0}^{1}(\Omega) .
$$

Using (3.2) and the trace theorem with scaling, we have

$$
\begin{aligned}
\sum_{e \in \mathcal{E}_{h}} \frac{1}{|e|}\left(\left\|\left\{\left\{\partial\left(\phi-\Pi_{h} \phi\right) / \partial n_{e}\right\}\right\}\right\|_{L_{2}(e)}^{2}+\sum_{e \in \mathcal{E}_{h}^{i}} \frac{1}{|e|}\left\|\llbracket \partial\left(\phi-\Pi_{h} \phi\right) / \partial n \rrbracket\right\|_{L_{2}(e)}^{2}\right. \\
+\sum_{e \in \mathcal{E}_{h}^{i}} \frac{1}{|e|}\left\|\partial\left(\phi-\Pi_{h} \phi\right) / \partial t_{e}\right\|_{L_{2}(e)}^{2} \leq C|\phi|_{H^{2}(\Omega)}^{2} \quad \forall \phi \in H^{2}(\Omega) \cap H_{0}^{1}(\Omega) .
\end{aligned}
$$

In view of (2.8), the estimates (3.2) and (3.3) also imply

$$
\left\|\Pi_{h} \phi\right\|_{h} \leq C|\phi|_{H^{2}(\Omega)} \quad \forall \phi \in H^{2}(\Omega) \cap H_{0}^{1}(\Omega) .
$$

The following result [13, Lemma 2.1 and Lemma 4.2] concerning the interpolant $\Pi_{h} \bar{y}$ of the optimal state will be needed in the analysis.

Lemma 3.1. There exists a positive constant $C$ independent of $h$ such that

$$
\begin{aligned}
& \left\|\bar{y}-\Pi_{h} \bar{y}\right\|_{h} \leq C h^{\tau}, \\
& \left.\sum_{e \in \mathcal{E}_{h}}|e|\|\| \partial^{2}\left(\Pi_{h} \bar{y}\right) / \partial n_{e}^{2}\right]\|\|_{L_{2}(e)}^{2} \leq C h^{2 \tau} .
\end{aligned}
$$

\subsection{An A Posteriori $L_{2}$ Error Estimate}

Here we derive an error estimate for $\left\|\bar{u}-\bar{u}_{h}\right\|_{L_{2}(\Omega)}$ in terms of $\left|\bar{u}_{h}\right|_{H^{1}(\Omega)}$.

Lemma 3.2. There exists a positive constant $C$ independent of $h$ such that

$$
\left\|\bar{u}-\bar{u}_{h}\right\|_{L_{2}(\Omega)} \leq C\left[h^{\tau}+h\left|\bar{u}_{h}\right|_{H^{1}(\Omega)}\right] .
$$

Proof. Let the function $\phi \in H_{0}^{1}(\Omega)$ be defined by

$$
(\nabla v, \nabla \phi)=\left(\bar{u}-\bar{u}_{h}, v\right) \quad \forall v \in H_{0}^{1}(\Omega) .
$$

Since $\Omega$ is convex, we have $\phi \in H^{2}(\Omega) \cap H_{0}^{1}(\Omega)$ and

$$
|\phi|_{H^{2}(\Omega)} \leq C|| \bar{u}-\bar{u}_{h} \|_{L_{2}(\Omega)} .
$$


It follows from (2.14), (2.15) and (3.8) that

$$
\begin{aligned}
\left(\bar{u}-\bar{u}_{h}, \bar{u}-\bar{u}_{h}\right)=\left(\nabla\left(\bar{u}-\bar{u}_{h}\right), \nabla \phi\right) & =(\nabla \bar{u}, \nabla \phi)-\left(\nabla \bar{u}_{h}, \nabla \Pi_{h} \phi\right)-\left(\nabla \bar{u}_{h}, \nabla\left(\phi-\Pi_{h} \phi\right)\right) \\
& =\int_{\Omega}\left(D^{2} \bar{y}: D^{2} \phi\right) d x-a_{h}\left(\bar{y}_{h}, \Pi_{h} \phi\right)-\left(\nabla \bar{u}_{h}, \nabla\left(\phi_{h}-\Pi_{h} \phi\right)\right),
\end{aligned}
$$

and from (3.2) and (3.9),

$$
\left|\left(\nabla \bar{u}_{h}, \nabla\left(\phi-\Pi_{h} \phi\right)\right)\right| \leq C h\left|\bar{u}_{h}\right|_{H^{1}(\Omega)}|| \bar{u}-\bar{u}_{h} \|_{L_{2}(\Omega)} .
$$

Furthermore it follows from (2.4) and (3.1) that

$$
\begin{aligned}
& \int_{\Omega}\left(D^{2} \bar{y}: D^{2} \phi\right) d x-a_{h}\left(\bar{y}_{h}, \Pi_{h} \phi\right) \\
& =\sum_{T \in \mathcal{T}_{h}} \int_{T}\left[D^{2}\left(\bar{y}-\bar{y}_{h}\right): D^{2} \phi\right] d x+\sum_{T \in \mathcal{T}_{h}} \int_{T}\left[D^{2} \bar{y}_{h}: D^{2}\left(\phi-\Pi_{h} \phi\right)\right] d x \\
& -\sum_{e \in \mathcal{E}_{h}^{i}} \int_{e}\left\{\left\{\partial^{2} \bar{y}_{h} / \partial n^{2}\right\}\right\} \llbracket \partial \Pi_{h} \phi / \partial n \rrbracket d s-\sum_{e \in \mathcal{E}_{h}^{i}} \int_{e}\left\{\left\lfloor\partial^{2} \Pi_{h} \phi / \partial n^{2}\right\} \llbracket \partial \bar{y}_{h} / \partial n \rrbracket d s\right. \\
& -\sum_{e \in \mathcal{E}_{h}^{i}} \frac{\sigma}{|e|} \int_{e} \llbracket \partial \bar{y}_{h} / \partial n \rrbracket \llbracket \partial \Pi_{h} \phi / \partial n \rrbracket d s \\
& \left.=\sum_{T \in \mathcal{T}_{h}} \int_{T}\left[D^{2}\left(\bar{y}-\bar{y}_{h}\right): D^{2} \phi\right] d x-\sum_{e \in \mathcal{E}_{h}} \int_{e} \llbracket \partial^{2} \bar{y}_{h} / \partial n_{e}^{2} \rrbracket\left\{\partial\left(\phi-\Pi_{h} \phi\right) / \partial n_{e}\right\}\right] d s \\
& -\sum_{e \in \mathcal{E}_{h}^{i}} \int_{e} \llbracket \partial^{2} \bar{y}_{h} / \partial t_{e} \partial n_{e} \rrbracket\left(\partial\left(\phi-\Pi_{h} \phi\right) / \partial t_{e}\right) d s-\sum_{e \in \mathcal{E}_{h}^{i}} \int_{e}\left\{\left\{\partial^{2} \Pi_{h} \phi / \partial n^{2}\right\} \llbracket \partial \bar{y}_{h} / \partial n \rrbracket d s\right. \\
& -\sum_{e \in \mathcal{E}_{h}^{i}} \frac{\sigma}{|e|} \int_{e} \llbracket \partial \bar{y}_{h} / \partial n \rrbracket \llbracket \partial \Pi_{h} \phi / \partial n \rrbracket d s \\
& =S_{1}+S_{2}+S_{3}+S_{4}+S_{5} \text {, }
\end{aligned}
$$

and the terms $S_{i}(1 \leq i \leq 5)$ can be estimated as follows:

$\left|S_{1}\right| \leq C h^{\tau}|\phi|_{H^{2}(\Omega)} \leq C h^{\tau}\left\|\bar{u}-\bar{u}_{h}\right\|_{L_{2}(\Omega)}$

by (2.8), (2.9) and (3.9);

$$
\begin{aligned}
& \left.\left|S_{2}\right| \leq\left(\sum_{e \in \mathcal{E}_{h}}|e|\|\| \partial^{2} \bar{y}_{h} / \partial n_{e}^{2}\right]\|\|_{L_{2}(e)}^{2}\right)^{\frac{1}{2}}\left(\sum_{e \in \mathcal{E}_{h}}|e|^{-1} \|\left\{\left\{\partial\left(\phi-\Pi_{h} \phi\right) / \partial n_{e}\right\}\|\|_{L_{2}(e)}^{2}\right)^{\frac{1}{2}}\right. \\
& \left.\left.\leq\left(2 \sum_{e \in \mathcal{E}_{h}}\left[|e|\|\| \partial^{2}\left(\bar{y}_{h}-\Pi_{h} \bar{y}\right) / \partial n_{e}^{2}\right]\|\|_{L_{2}(e)}^{2}+|e|\|\| \partial^{2} \Pi_{h} \bar{y} / \partial n_{e}^{2}\right]\|\|_{L_{2}(e)}^{2}\right]\right)^{\frac{1}{2}}\left\|\bar{u}-\bar{u}_{h}\right\|_{L_{2}(\Omega)} \\
& \left.\leq C\left(\sum_{T \in \mathcal{T}_{h}}\left|\bar{y}_{h}-\Pi_{h} \bar{y}\right|_{H^{2}(T)}^{2}+\sum_{e \in \mathcal{E}_{h}}|e|\|\| \partial^{2} \Pi_{h} \bar{y} / \partial n_{e}^{2}\right]\|\|_{L_{2}(e)}^{2}\right)^{\frac{1}{2}}\left\|\bar{u}-\bar{u}_{h}\right\|_{L_{2}(\Omega)} \\
& \leq C\left(\sum_{T \in \mathcal{T}_{h}}\left[\left|\bar{y}_{h}-\bar{y}\right|_{H^{2}(T)}^{2}+\left|\bar{y}-\Pi_{h} \bar{y}\right|_{H^{2}(T)}^{2}\right]+\sum_{e \in \mathcal{E}_{h}}|e|\|\|\left[\partial^{2} \Pi_{h} \bar{y} / \partial n_{e}^{2}\right]\|\|_{L_{2}(e)}^{2}\right)^{\frac{1}{2}}\left\|\bar{u}-\bar{u}_{h}\right\|_{L_{2}(\Omega)} \leq C h^{\tau}\left\|\bar{u}-\bar{u}_{h}\right\|_{L_{2}(\Omega)}
\end{aligned}
$$

by (2.8), (2.9), (3.3), Lemma 3.1, (3.9), and a standard inverse/discrete estimate;

$$
\left.\left|S_{3}\right| \leq\left(\sum_{e \in \mathcal{E}_{h}^{i}}|e|\|\| \partial^{2} \bar{y}_{h} / \partial t_{e} \partial n_{e}\right]\|\|_{L_{2}(e)}^{2}\right)^{\frac{1}{2}}\left(\sum_{e \in \mathcal{E}_{h}^{i}}|e|^{-1}\left\|\partial\left(\phi-\Pi_{h} \phi\right) / \partial t_{e}\right\|_{L_{2}(e)}^{2}\right)^{\frac{1}{2}}
$$




$$
\begin{aligned}
& \leq C\left(\sum_{e \in \mathcal{E}_{h}^{i}}|e|^{-1}\left\|\llbracket \partial \bar{y}_{h} / \partial n_{e} \rrbracket\right\|_{L_{2}(e)}^{2}\right)^{\frac{1}{2}}\left\|\bar{u}-\bar{u}_{h}\right\|_{L_{2}(\Omega)} \\
& =C\left(\sum_{e \in \mathcal{E}_{h}^{i}}|e|^{-1}\left\|\llbracket \partial\left(\bar{y}_{h}-\bar{y}\right) / \partial n_{e} \rrbracket\right\|_{L_{2}(e)}^{2}\right)^{\frac{1}{2}}\left\|\bar{u}-\bar{u}_{h}\right\|_{L_{2}(\Omega)} \leq C h^{\tau}\left\|\bar{u}-\bar{u}_{h}\right\|_{L_{2}(\Omega)}
\end{aligned}
$$

by (2.8), (2.9), (3.3), (3.9) and a standard inverse estimate;

$$
\begin{aligned}
\left|S_{4}\right| & \leq\left(\sum_{e \in \mathcal{E}_{h}^{i}}|e|\left\|\left\{\left\{\partial^{2} \Pi_{h} \phi / \partial n^{2}\right\}\right\rangle\right\|_{L_{2}(e)}^{2}\right)^{\frac{1}{2}}\left(\sum_{e \in \mathcal{E}_{h}^{i}}|e|^{-1}\left\|\llbracket \partial \partial\left(\bar{y}_{h}-\bar{y}\right) / \partial n \rrbracket\right\|_{L_{2}(e)}^{2}\right)^{\frac{1}{2}} \\
& \leq C h^{\tau}\left(\sum_{T \in \mathcal{T}_{h}}\left|\Pi_{h} \phi\right|_{H^{2}(T)}^{2}\right)^{\frac{1}{2}} \leq C h^{\tau}|\phi|_{H^{2}(\Omega)} \leq C h^{\tau}\left\|\bar{u}-\bar{u}_{h}\right\|_{L_{2}(\Omega)}
\end{aligned}
$$

by (2.8), (2.9), (3.4), (3.9) and a standard inverse estimate;

$$
\left|S_{5}\right| \leq \sigma\left(\sum_{e \in \mathcal{E}_{h}^{i}}|e|^{-1}\left\|\llbracket \partial\left(\bar{y}_{h}-\bar{y}\right) / \partial n \rrbracket\right\|_{L_{2}(e)}^{2}\right)^{\frac{1}{2}}\left(\sum_{e \in \mathcal{E}_{h}^{i}}|e|^{-1}\left\|\llbracket \partial\left(\phi-\Pi_{h} \phi\right) / \partial n \rrbracket\right\|_{L_{2}(e)}^{2}\right)^{\frac{1}{2}} \leq C h^{\tau}\left\|\bar{u}-\bar{u}_{h}\right\|_{L_{2}(\Omega)}
$$

by (2.8), (2.9), (3.3) and (3.9).

The estimate (3.7) follows from (3.10)-(3.12) and the estimates for $S_{i}(1 \leq i \leq 5)$.

\subsection{An A Priori $L_{2}$ Error Estimate}

We can turn the estimate (3.7) into an a priori error estimate by providing a bound for $\left|\bar{u}_{h}\right|_{H^{1}(\Omega)}$, which in turn requires a bound on the nonnegative measure $\bar{\lambda}_{h}$ defined by (2.17).

Lemma 3.3. There exists a positive constant $C$ independent of $h$ such that $\bar{\lambda}_{h}(\Omega) \leq C$.

Proof. According to (2.16b), the coefficient $c_{h, p}=0$ if $\psi(p)>\bar{y}_{h}(p)$. Since $\bar{y}_{h}$ converges to $\bar{y}$ uniformly on $\Omega$ by (2.10) and $\bar{y}<\psi$ in a neighborhood of $\partial \Omega$, there exists a compact subset $\mathfrak{C}$ of $\Omega$ such that $\bar{\lambda}_{h}(\Omega \backslash \mathfrak{C})=0$ for $h$ sufficiently small.

Let $\phi \in C_{c}^{\infty}(\Omega)$ such that $\phi=1$ on an open neighborhood of $\mathfrak{C}$. For sufficiently small $h$, it follows from (2.9), (2.18), (3.2) and (3.4) that

$$
\begin{aligned}
\bar{\lambda}_{h}(\Omega)=\int_{\Omega}\left(\Pi_{h} \phi\right) d \bar{\lambda}_{h} & =\left(y_{d}-\bar{y}_{h}, \Pi_{h} \phi\right)-\beta a_{h}\left(\bar{y}_{h}, \Pi_{h} \phi\right) \\
& \leq\left\|y_{d}-\bar{y}_{h}\right\|_{L_{2}(\Omega)}\left\|\Pi_{h} \phi\right\|_{L_{2}(\Omega)}+C\left\|\bar{y}_{h}\right\|_{h}\left\|\Pi_{h} \phi\right\|_{h} \leq C\left(\left\|y_{d}-\bar{y}_{h}\right\|_{L_{2}(\Omega)}+\left\|\bar{y}_{h}\right\|_{h}\right)|\phi|_{H^{2}(\Omega)} \leq C .
\end{aligned}
$$

Lemma 3.4. There exists a positive constant $C$ independent of $h$ such that

$$
\left|\bar{u}_{h}\right|_{H^{1}(\Omega)} \leq C\left(1+|\ln h|^{\frac{1}{2}}\right) .
$$

Proof. It follows from (2.9), (2.19) and Lemma 3.3 that

$$
\left(\nabla \bar{u}_{h}, \nabla \bar{u}_{h}\right)=\frac{1}{\beta}\left[\left(y_{d}-\bar{y}_{h}, \bar{u}_{h}\right)-\int_{\Omega} \bar{u}_{h} d \bar{\lambda}_{h}\right] \leq C\left(\left\|\bar{u}_{h}\right\|_{L_{2}(\Omega)}+\bar{\lambda}_{h}(\Omega)\left\|\bar{u}_{h}\right\|_{L_{\infty}(\Omega)}\right) \leq C\left(1+|\ln h|^{\frac{1}{2}}\right)\left|\bar{u}_{h}\right|_{H^{1}(\Omega)},
$$

where we have used a Poincaré-Friedrichs inequality and a discrete Sobolev inequality [9, Section 4.9].

Putting Lemma 3.2 and Lemma 3.4 together, we have the following theorem. 
Theorem 3.5. There exists a positive constant $C$ independent of $h$ such that

$$
\left\|\bar{u}-\bar{u}_{h}\right\|_{L_{2}(\Omega)} \leq C\left[h^{\tau}+h\left(1+|\ln h|^{\frac{1}{2}}\right)\right]
$$

In the case of quasi-uniform meshes, by taking advantage of the availability of global inverse estimates, we have the following result that slightly improves (3.14) when $\alpha=1$.

Theorem 3.6. In the case where $\mathcal{T}_{h}$ is a quasi-uniform triangulation of $\Omega$, we have

$$
\left\|\bar{u}-\bar{u}_{h}\right\|_{L_{2}(\Omega)} \leq C h^{\alpha}
$$

Proof. In view of (3.7) with $\tau=\alpha$, it suffices to show that

$$
\left|\bar{u}_{h}\right|_{H^{1}(\Omega)} \leq C h^{-1+\alpha} .
$$

It follows from (2.4) and (3.1) that

$$
\begin{aligned}
a\left(\bar{y}_{h}, v\right)= & \sum_{T \in \mathcal{T}_{h}} \int_{T}\left(D^{2} \bar{y}_{h}: D^{2} v\right) d x+\sum_{e \in \mathcal{E}_{h}^{i}} \int_{e}\left\{\left\{\partial^{2} \bar{y}_{h} / \partial n^{2}\right\}\right\rfloor \partial v / \partial n \rrbracket d s \\
& +\sum_{e \in \mathcal{E}_{h}^{i}} \int_{e}\left\{\left\{\partial^{2} v / \partial n^{2}\right\}\right\} \llbracket \partial \bar{y}_{h} / \partial n \rrbracket d s+\sum_{e \in \mathcal{E}_{h}^{i}} \frac{\sigma}{|e|} \int_{e} \llbracket \partial \bar{y}_{h} / \partial n \rrbracket \llbracket \partial v / \partial n \rrbracket d s \\
= & -\sum_{e \in \mathcal{E}_{h}} \int_{e} \llbracket\left\{\partial^{2} \bar{y}_{h} / \partial n_{e}^{2} \rrbracket \llbracket\left\{\partial v / \partial n_{e}\right\} d s-\sum_{e \in \mathcal{E}_{h}^{i}} \int_{e} \llbracket \mid \partial^{2} \bar{y}_{h} / \partial t_{e} \partial n_{e} \rrbracket\left(\partial v / \partial t_{e}\right) d s\right. \\
& +\sum_{e \in \mathcal{E}_{h}^{i}} \int_{e}\left\{\left\{\partial^{2} v / \partial n^{2}\right\}\right\} \llbracket \partial \bar{y}_{h} / \partial n \rrbracket d s+\sum_{e \in \mathcal{E}_{h}^{i}} \frac{\sigma}{|e|} \int_{e} \llbracket \partial \bar{y}_{h} / \partial n \rrbracket \llbracket \partial v / \partial n \rrbracket d s \\
= & R_{1}+R_{2}+R_{3}+R_{4} .
\end{aligned}
$$

Similar to the treatment of the terms on the right-hand side of (3.12), we can estimate $R_{i}(1 \leq i \leq 4)$ by Theorem 2.3 and global inverse estimates (where the quasi-uniformity of $\mathcal{T}_{h}$ is crucial) as follows:

$$
\begin{aligned}
& \left|R_{1}\right| \leq\left(\sum_{e \in \mathcal{E}_{h}}|e|\left\|\mid \llbracket \partial^{2} \bar{y}_{h} / \partial n_{e}^{2} \rrbracket\right\|_{L_{2}(e)}^{2}\right)^{\frac{1}{2}}\left(\sum_{e \in \mathcal{E}_{h}}|e|^{-1} \|\left\{\left\{\partial v / \partial n_{e}\right\} \|_{L_{2}(e)}^{2}\right)^{\frac{1}{2}} \leq C h^{\alpha}\left[h^{-1}|v|_{H^{1}(\Omega)}\right]=C h^{-1+\alpha}|v|_{H^{1}(\Omega)},\right. \\
& \left|R_{2}\right| \leq\left(\sum_{e \in \mathcal{E}_{h}^{i}} \mid e\|\|\left\|\partial^{2} \bar{y}_{h} / \partial t_{e} \partial n_{e} \rrbracket\right\| \|_{L_{2}(e)}^{2}\right)^{\frac{1}{2}}\left(\sum_{e \in \mathcal{E}_{h}^{i}}|e|^{-1}\left\|\partial v / \partial t_{e}\right\|_{L_{2}(e)}^{2}\right)^{\frac{1}{2}} \leq C h^{\alpha}\left[h^{-1}|v|_{H^{1}(\Omega)}\right]=C h^{-1+\alpha}|v|_{H^{1}(\Omega)}, \\
& \left.\left|R_{3}\right| \leq\left(\sum_{e \in \mathcal{E}_{h}^{i}}|e| \| \mid\left\{\partial^{2} v / \partial n^{2}\right\}\right\} \|_{L_{2}(e)}^{2}\right)^{\frac{1}{2}}\left(\sum_{e \in \mathcal{E}_{h}^{i}}|e|^{-1}\left\|\llbracket \partial\left(\bar{y}_{h}-\bar{y}\right) / \partial n \rrbracket\right\|_{L_{2}(e)}^{2}\right)^{\frac{1}{2}} \leq C\left[h^{-1}|v|_{H^{1}(\Omega)}\right] h^{\alpha}=C h^{-1+\alpha}|v|_{H^{1}(\Omega)}, \\
& \left|R_{4}\right| \leq \sigma\left(\sum_{e \in \mathcal{E}_{h}^{i}}|e|^{-1}\left\|\llbracket \partial\left(\bar{y}_{h}-\bar{y}\right) / \partial n \rrbracket\right\|_{L_{2}(e)}^{2}\right)^{\frac{1}{2}}\left(\sum_{e \in \mathcal{E}_{h}^{i}}|e|^{-1}\|\llbracket \partial v / \partial n \rrbracket\|_{L_{2}(e)}^{2}\right)^{\frac{1}{2}} \leq C h^{\alpha}\left[h^{-1}|v|_{H^{1}(\Omega)}\right]=C h^{-1+\alpha}|v|_{H^{1}(\Omega)} .
\end{aligned}
$$

The estimate (3.16) follows from (2.15), (3.17) and the estimates for $R_{i}(1 \leq i \leq 4)$.

Combining (3.15) and (3.16), we immediately have the following corollary.

Corollary 3.7. In the case where $\alpha=1$ and $\mathcal{T}_{h}$ is a quasi-uniform triangulation of $\Omega$, the approximate optimal control $\bar{u}_{h}$ converges weakly in $H^{1}(\Omega)$ to the optimal control $\bar{u}$. 


\subsection{Convergence of $\bar{\lambda}_{h}$ to $\bar{\lambda}$}

As a byproduct of the analysis in Section 3.2 and Section 3.3, we can deduce the convergence of $\bar{\lambda}_{h}$ to $\bar{\lambda}_{\text {in }}$ the dual space of $H^{2}(\Omega) \cap H_{0}^{1}(\Omega)$.

Theorem 3.8. Let $L$ and $L_{h}$ be defined by

$$
L(\phi)=\int_{\Omega} \phi d \bar{\lambda} \quad \text { and } \quad L_{h}(\phi)=\int_{\Omega} \phi d \bar{\lambda}_{h} \quad \forall \phi \in H^{2}(\Omega) \cap H_{0}^{1}(\Omega) .
$$

There exists a constant $C$ independent of $h$ such that

$$
\left\|L-L_{h}\right\|_{\left[H^{2}(\Omega) \cap H_{0}^{1}(\Omega)\right]^{\prime}} \leq C h^{\tau} .
$$

Proof. Let $\phi \in H^{2}(\Omega) \cap H_{0}^{1}(\Omega)$ be arbitrary. It follows from (1.3), (1.5a) and (2.18) that

$$
\begin{aligned}
\int_{\Omega} \phi d \bar{\lambda}-\int_{\Omega} \Pi_{h} \phi d \bar{\lambda}_{h} & =\left(y_{d}, \phi\right)-\mathcal{A}(\bar{y}, \phi)-\left[\left(y_{d}, \Pi_{h} \phi\right)-\mathcal{A}_{h}\left(\bar{y}_{h}, \Pi_{h} \phi\right)\right] \\
& =\left(y_{d}, \phi-\Pi_{h} \phi\right)-\beta\left[\int_{\Omega}\left(D^{2} \bar{y}: D^{2} \phi\right) d x-a_{h}\left(\bar{y}_{h}, \Pi_{h} \phi\right)\right]+\left[\left(\bar{y}_{h}, \Pi_{h} \phi\right)-(\bar{y}, \phi)\right] \\
& =T_{1}+T_{2}+T_{3},
\end{aligned}
$$

and we have

$$
\left|T_{1}\right| \leq\left\|y_{d}\right\|_{L_{2}(\Omega)}\left\|\phi-\Pi_{h} \phi\right\|_{L_{2}(\Omega)} \leq C h^{2}|\phi|_{H^{2}(\Omega)}
$$

by (3.2),

$$
\left|T_{2}\right| \leq C h^{\tau}|\phi|_{H^{2}(\Omega)}
$$

by the estimate for (3.12),

$$
\left|T_{3}\right| \leq\left|\left(\bar{y}_{h}-\bar{y}, \Pi_{h} \phi\right)+\left(\bar{y}, \Pi_{h} \phi-\phi\right)\right| \leq C h^{\tau}|\phi|_{H^{2}(\Omega)}
$$

by (2.9) and (3.2). It follows that

$$
\left|\int_{\Omega} \phi d \bar{\lambda}-\int_{\Omega} \Pi_{h} \phi d \bar{\lambda}_{h}\right| \leq C h^{\tau}|\phi|_{H^{2}(\Omega)} .
$$

On the other hand Lemma 3.3 and a standard interpolation error estimate [9] imply

$$
\left|\int_{\Omega}\left(\phi-\Pi_{h} \phi\right) d \bar{\lambda}_{h}\right| \leq\left\|\phi-\Pi_{h} \phi\right\|_{L_{\infty}(\Omega)} \bar{\lambda}_{h}(\Omega) \leq C h|\phi|_{H^{2}(\Omega)} .
$$

Combining (3.18) and (3.19), we have

$$
\left|\int_{\Omega} \phi d \bar{\lambda}-\int_{\Omega} \phi d \bar{\lambda}_{h}\right| \leq C h^{\tau}|\phi|_{H^{2}(\Omega)} \quad \forall \phi \in H^{2}(\Omega) \cap H_{0}^{1}(\Omega) .
$$

The weak convergence of $\bar{\lambda}_{h}$ to $\bar{\lambda}$ in the dual space of $C(\bar{\Omega})$ follows as a corollary.

Corollary 3.9. We have

$$
\lim _{h \rightarrow 0} \int_{\Omega} \zeta d \bar{\lambda}_{h}=\int_{\Omega} \zeta d \bar{\lambda} \quad \forall \zeta \in C(\bar{\Omega}) .
$$


Proof. Let $\left\{h_{n}\right\}_{n=1}^{\infty}$ be a sequence of positive numbers that converge to 0. It follows from Lemma 3.3 and Helly's Theorem [31, Theorem 10.12] that there exist a subsequence $\left\{h_{n_{k}}\right\}_{k=1}^{\infty}$ and a nonnegative Borel measure $\mu$ such that

$$
\lim _{k \rightarrow \infty} \int_{\Omega} \zeta d \bar{\lambda}_{h_{n_{k}}}=\int_{\Omega} \zeta d \mu \quad \forall \zeta \in C(\bar{\Omega})
$$

It only remains to show that $\mu=\bar{\lambda}$.

Let $\mathfrak{C}$ be the compact subset defined in the proof of Lemma 3.3. Then we have $\mu(\Omega \backslash \mathfrak{C})=0$ by (3.21). Furthermore we can also assume that $\bar{\lambda}(\Omega \backslash \mathfrak{C})=0$, since the constraint set $K$ in (1.2) is disjoint from $\partial \Omega$. Therefore it suffices to show that

$$
\int_{\Omega} \zeta d \bar{\lambda}=\int_{\Omega} \zeta d \mu
$$

for all $\zeta$ in the space $C_{\Omega}(\Omega)=\{\zeta \in C(\bar{\Omega}): \operatorname{supp} \zeta \subset \Omega\}$, where $\Omega$ is a compact subset of $\Omega$ whose interior contains $\mathcal{C}$.

Observe that Theorem 3.8 and (3.21) imply

$$
\int_{\Omega} \zeta d \bar{\lambda}=\int_{\Omega} \zeta d \mu \quad \forall \zeta \in C_{c}^{\infty}(\Omega) .
$$

Since every function in $C_{\Omega}(\Omega)$ is the limit in $C(\bar{\Omega})$ of a sequence of functions in $C_{c}^{\infty}(\Omega)$, the relation (3.22) follows from (3.23).

\subsection{Convergence of $\bar{u}_{h}$ in the $H^{1}$ Norm}

The following result relates the convergence of $\bar{u}_{h}$ in the $H^{1}$ norm to the convergence of $\bar{\lambda}_{h}$.

Theorem 3.10. We have

$$
\lim _{h \rightarrow 0}\left|\bar{u}-\bar{u}_{h}\right|_{H^{1}(\Omega)}=0 \quad \text { if and only if } \quad \limsup _{h \rightarrow 0}\left|\int_{v \in V_{h}} v d \bar{\lambda}_{h}-\int_{\Omega} v d \bar{\lambda}\right| /|v|_{H^{1}(\Omega)}=0 .
$$

Proof. Since $H^{2}(\Omega) \cap H_{0}^{1}(\Omega)$ is dense in $H_{0}^{1}(\Omega)$ and $\bar{u}=-\Delta \bar{y} \in H_{0}^{1}(\Omega)$, it follows from (1.3), (1.5a) and integration by parts that

$$
\int_{\Omega} v d \bar{\lambda}=\left(y_{d}-\bar{y}, v\right)-\beta(\nabla \bar{u}, \nabla v) \quad \forall v \in H_{0}^{1}(\Omega)
$$

Let $R_{h} \bar{u} \in V_{h}$ be the Ritz projection of $\bar{u}$, i.e.,

$$
\left(\nabla R_{h} \bar{u}, \nabla v\right)=(\nabla \bar{u}, \nabla v) \quad \forall v \in V_{h} .
$$

Let $v \in V_{h}$ be arbitrary. It follows from (2.19) and (3.25) that

$$
\left(\nabla\left(R_{h} \bar{u}-\bar{u}_{h}\right), \nabla v\right)=\left(\nabla\left(\bar{u}-\bar{u}_{h}\right), \nabla v\right)=\frac{1}{\beta}\left[\left(\bar{y}_{h}-\bar{y}, v\right)+\left(\int_{\Omega} v d \bar{\lambda}_{h}-\int_{\Omega} v d \bar{\lambda}\right)\right],
$$

and hence, by a Poincaré-Friedrichs inequality,

$$
\left|R_{h} \bar{u}-\bar{u}_{h}\right|_{H^{1}(\Omega)}=\sup _{v \in V_{h}} \frac{\left(\nabla\left(R_{h} \bar{u}-\bar{u}_{h}\right), \nabla v\right)}{|v|_{H^{1}(\Omega)}} \leq C \| \bar{y}-\left.\bar{y}_{h}\right|_{L_{2}(\Omega)}+\frac{1}{\beta} \sup _{v \in V_{h}}\left|\int_{\Omega} v d \bar{\lambda}_{h}-\int_{\Omega} v d \bar{\lambda}\right| /|v|_{H^{1}(\Omega)} .
$$

Similarly we have

$$
\frac{1}{\beta} \sup _{v \in V_{h}}\left|\int_{\Omega} v d \bar{\lambda}_{h}-\int_{\Omega} v d \bar{\lambda}\right| /|v|_{H^{1}(\Omega)} \leq\left|R_{h} \bar{u}-\bar{u}_{h}\right|_{H^{1}(\Omega)}+C|| \bar{y}-\bar{y}_{h} \|_{L_{2}(\Omega)} .
$$

It follows that

$$
\left|\bar{u}-\bar{u}_{h}\right|_{H^{1}(\Omega)} \leq\left|\bar{u}-R_{h} \bar{u}\right|_{H^{1}(\Omega)}+\left|R_{h} \bar{u}-\bar{u}_{h}\right|_{H^{1}(\Omega)}
$$




$$
\leq\left|\bar{u}-R_{h} \bar{u}_{H^{1}(\Omega)}+C\right| \bar{y}-\bar{y}_{h} \|_{L_{2}(\Omega)}+\frac{1}{\beta} \sup _{v \in V_{h}}\left|\int_{\Omega} v d \bar{\lambda}_{h}-\int_{\Omega} v d \bar{\lambda}\right| /|v|_{H^{1}(\Omega)},
$$

and

$$
\begin{aligned}
\frac{1}{\beta} \sup _{v \in V_{h}}\left|\int_{\Omega} v d \bar{\lambda}_{h}-\int_{\Omega} v d \bar{\lambda}\right| /|v|_{H^{1}(\Omega)} & \leq\left|R_{h} \bar{u}-\bar{u}_{h}\right|_{H^{1}(\Omega)}+C\left\|\bar{y}-\bar{y}_{h}\right\|_{L_{2}(\Omega)} \\
& \leq\left|\bar{u}-\bar{u}_{h}\right|_{H^{1}(\Omega)}+\left|\bar{u}-R_{h} \bar{u}\right|_{H^{1}(\Omega)}+C|| \bar{y}-\bar{y}_{h} \|_{L_{2}(\Omega)} .
\end{aligned}
$$

In view of the limits

$$
\lim _{h \rightarrow 0}\left\|\bar{y}-\bar{y}_{h}\right\|_{L_{2}(\Omega)}=0 \quad \text { and } \quad \lim _{h \rightarrow 0}\left|\bar{u}-R_{h} \bar{u}\right|_{H^{1}(\Omega)}=\lim _{h \rightarrow 0} \inf _{v \in V_{h}}|\bar{u}-v|_{H^{1}(\Omega)}=0,
$$

the relation (3.24) follows from (3.26) and (3.27).

\section{Numerical Results}

In this section we report some numerical results that illustrate the performance of the three post-processing procedures and confirm the theoretical results.

Even though the a priori error estimates (2.11), (2.13) and (3.14) for the outputs of these procedures are similar, numerical results indicate that the third procedure has some advantages over the other two, and the second procedure also has some advantages over the first. The computational cost for the first procedure is the lowest. The computational cost for the second procedure is higher because it requires the solution of a system of equations involving the (wellconditioned) mass matrix. The computational cost for the third procedure is the highest since it requires the solution of a system of equations involving the (ill-conditioned) stiffness matrix for a Poisson problem. However these differences are not significant in the overall picture since the cost for computing $\bar{y}_{h}$ is much higher.

The discrete problem (2.6) in the following examples are solved by a primal-dual active set algorithm [27]. The discrete state associated with the mesh at refinement level $j$ is denoted by $\bar{y}_{j}$ and the corresponding discrete control is denoted by $\bar{u}_{j}$. We use uniform meshes in Example 1-Example 3 and a graded mesh in Example 4. Based on our experience, we take the penalty parameter $\sigma$ to be 5 for uniform meshes. Since the triangles in a graded mesh are less regular, we take $\sigma$ to be 20 for Example 4.

\section{Example 1}

In this example we consider a problem with a known solution to validate the numerical computations of the three post-processing procedures. We begin with the optimal control problem (1.1) (or equivalently (1.2)) on the disc $\{x:|x|<2\}$ with the data $\beta=1, \psi(x)=|x|^{2}-1$, and

$$
y_{d}(x)= \begin{cases}C_{1}|x|^{2} \ln |x|+C_{2}|x|^{2}+C_{3} \ln |x|+C_{4}+\frac{1}{32}|x|^{4}+2 & |x|>r_{0} \\ |x|^{2}-1 & |x| \leqslant r_{0},\end{cases}
$$

where $r_{0} \approx 0.19744467, C_{1} \approx-0.64571931, C_{2} \approx 0.59329783, C_{3} \approx-0.02498301, C_{4} \approx-1.06556035$. This problem can be solved exactly because of rotational symmetry, and the exact solution is given by

$$
\bar{y}(x)=\left\{\begin{array}{ll}
y_{d}-2 & |x|>r_{0} \\
|x|^{2}-1 & |x| \leqslant r_{0}
\end{array} .\right.
$$

Now we restrict the problem to the domain $\Omega=(-0.5,0.5)^{2}$ with the same data so that the exact solution is the restriction of $\bar{y}$ on $\Omega$. Note that in this case we have nonhomogeneous boundary conditions on $\partial \Omega$ and we are solving a fourth order variational inequality that no longer corresponds to an optimal control problem. Nevertheless the quadratic $C^{0}$ interior penalty method can be applied to this problem with straightforward modifications. The error estimates remain the same (cf. [11,13]) and the post-processing procedures can also be modified accordingly.

The $L_{2}$ errors for the three post-processing procedures are reported in Table 4.1, from which we observe that all three procedures converge. The performance of Procedure 1 is as predicted by (2.11), since the elliptic regularity index 
Table 4.1: $L_{2}$ control errors for Example 1

\begin{tabular}{|c|c|c|c|c|c|c|}
\hline & \multicolumn{2}{|c|}{ Procedure 1} & \multicolumn{2}{|c|}{ Procedure 2} & \multicolumn{2}{|c|}{ Procedure 3} \\
\hline & $\left\|\bar{u}_{8}\right\|_{L_{2}(\Omega)}=2$ & 6088 & $\left\|\bar{u}_{8}\right\|_{L_{2}(\Omega)}=$ & 6088 & $\left\|\bar{u}_{8}\right\|_{L_{2}(\Omega)}=2$ & 6089 \\
\hline$j$ & $\frac{\left\|e_{u, j}\right\|_{L_{2}(\Omega)}}{\left\|\bar{u}_{8}\right\|_{L_{2}(\Omega)}}$ & order & $\frac{\left\|e_{u, j}\right\|_{L_{2}(\Omega)}}{\left\|\bar{u}_{8}\right\|_{L_{2}(\Omega)}}$ & order & $\frac{\left\|e_{u, j}\right\|_{L_{2}(\Omega)}}{\left\|\bar{u}_{8}\right\|_{L_{2}(\Omega)}}$ & order \\
\hline 1 & $3.9199 \mathrm{~B}$ & & 2.8541E-01 & & 2.0166 & \\
\hline 2 & $1.5122 \mathrm{E}$ & 1.37 & 1.1592 & 1.30 & 8.4087 & 1.26 \\
\hline 3 & $5.4681 \mathrm{E}-02$ & 1.47 & 4.4673 & 1.38 & 3.5872 & 1.23 \\
\hline 4 & $2.1361 \mathrm{~F}$ & 1.36 & 1.3958 & 1. & 9.8104 & 1.87 \\
\hline 5 & 9.6163E-03 & 1.15 & 5.4642 & 1.35 & 3.6688 & 1.42 \\
\hline 6 & 4.1432E-03 & 1.21 & $2.1095 \mathrm{E}$ & 1.37 & $1.3411 \mathrm{E}-03$ & 1.45 \\
\hline 7 & $1.8259 \mathrm{E}-03$ & 1.18 & $7.2998 \mathrm{E}-04$ & 1.53 & $4.2262 \mathrm{E}-04$ & 1.67 \\
\hline 8 & 8.5179E-04 & 1.10 & $2.6966 \mathrm{E}-04$ & 1.44 & $1.6245 \mathrm{E}-04$ & 1.38 \\
\hline
\end{tabular}

$\alpha=1$ for rectangular domains. The errors for Procedure 2 and Procedure 3 are $O\left(h^{1.5}\right)$, better than those predicted by (2.13) and (3.14). Procedure 3 also has slightly smaller errors than Procedure 2.

The observed convergence for $\bar{u}_{h, 2}$ and $\bar{u}_{h, 3}$ in the $L_{2}$ norm can be explained as follows. The solution $\bar{y}$ given by (4.1) is piecewise smooth and globally $C^{2}$. Hence $\bar{u}=-\Delta \bar{y}$ is piecewise smooth and globally continuous, which means that $\bar{u}$ belongs to $H^{(3 / 2)-\epsilon}(\Omega)$ for any $\epsilon>0$. The outputs $\bar{u}_{h, 2}$ and $\bar{u}_{h, 3}$, which belong to the quadratic Lagrange finite element space $V_{h}$, can therefore take advantage of this additional regularity and any superconvergence effects due to uniform meshing. On the other hand, since $\bar{u}_{h, 1}$ is a piecewise constant function, the $L_{2}$ error $\left\|\bar{u}-\bar{u}_{h, 1}\right\|_{L_{2}(\Omega)}$ remains $O(h)$.

We also report the $H^{1}$ errors for Procedure 2 and Procedure 3 in Table 4.2, which are both of magnitude $O\left(h^{0.5}\right)$. The observed $H^{1}$ errors for $\bar{u}_{h, i}(i=2,3)$ follow from the observed $L_{2}$ errors and the estimate

$$
\begin{aligned}
\left|\bar{u}-\bar{u}_{h, i}\right|_{H^{1}(\Omega)} & \leq\left|\bar{u}-\Pi_{h} \bar{u}\right|_{H^{1}(\Omega)}+\left|\Pi_{h} \bar{u}-\bar{u}_{h, i}\right|_{H^{1}(\Omega)} \\
& \leq\left|\bar{u}-\Pi_{h} \bar{u}\right|_{H^{1}(\Omega)}+C h^{-1} \| \Pi_{h} \bar{u}-\left.\bar{u}_{h, i}\right|_{L_{2}(\Omega)} \\
& \leq\left|\bar{u}-\Pi_{h} \bar{u}\right|_{H^{1}(\Omega)}+C h^{-1}\left[\left\|\Pi_{h} \bar{u}-\bar{u}\right\|_{L_{2}(\Omega)}+\left\|\bar{u}-\bar{u}_{h, i}\right\|_{L_{2}(\Omega)}\right],
\end{aligned}
$$

where we are able to apply a standard inverse estimate since $\mathcal{T}_{h}$ is quasi-uniform. We also observe that Procedure 3 provides a better approximation and the $H^{1}$ semi-norms of $u_{h, 3}$ are uniformly bounded, which agrees with (3.16).

Table 4.2: $H^{1}$ control errors for Example 1

\begin{tabular}{|c|ccc|ccc|}
\hline & \multicolumn{3}{|c|}{ Procedure 2} & \multicolumn{3}{c|}{ Procedure 3 } \\
\hline$j$ & $\left|\bar{u}_{j}\right|_{H^{1}(\Omega)}$ & $\frac{\left|e_{u, j}\right|_{H^{1}(\Omega)}}{\left|\bar{u}_{8}\right|_{H^{1}(\Omega)}}$ & order & $\left|\bar{u}_{j}\right|_{H^{1}(\Omega)}$ & $\frac{\left|e_{u, j}\right|_{H^{1}(\Omega)}}{\left|\bar{u}_{8}\right|_{H^{1}(\Omega)}}$ & order \\
\hline 1 & $1.1829 \mathrm{E}+01$ & $1.8579 \mathrm{E}+00$ & & $8.6859 \mathrm{E}+00$ & $1.0442 \mathrm{E}+00$ & \\
2 & $1.2166 \mathrm{E}+01$ & $1.6648 \mathrm{E}+00$ & 0.16 & $7.4953 \mathrm{E}+00$ & $7.1235 \mathrm{E}-01$ & 0.55 \\
3 & $9.2143 \mathrm{E}+00$ & $1.1079 \mathrm{E}+00$ & 0.59 & $6.8084 \mathrm{E}+00$ & $5.2240 \mathrm{E}-01$ & 0.45 \\
4 & $7.6552 \mathrm{E}+00$ & $7.3974 \mathrm{E}-01$ & 0.58 & $6.4549 \mathrm{E}+00$ & $2.6791 \mathrm{E}-01$ & 0.96 \\
5 & $6.9593 \mathrm{E}+00$ & $5.1118 \mathrm{E}-01$ & 0.53 & $6.3418 \mathrm{E}+00$ & $1.9737 \mathrm{E}-01$ & 0.44 \\
6 & $6.5935 \mathrm{E}+00$ & $3.6556 \mathrm{E}-01$ & 0.48 & $6.2819 \mathrm{E}+00$ & $1.5249 \mathrm{E}-01$ & 0.37 \\
7 & $6.4350 \mathrm{E}+00$ & $2.6297 \mathrm{E}-01$ & 0.48 & $6.2740 \mathrm{E}+00$ & $1.0535 \mathrm{E}-01$ & 0.53 \\
8 & $6.3471 \mathrm{E}+00$ & $1.8583 \mathrm{E}-01$ & 0.50 & $6.2664 \mathrm{E}+00$ & $7.7763 \mathrm{E}-02$ & 0.44 \\
\hline
\end{tabular}

For this example the exact coincidence set is the closed disc $\left\{x:|x| \leq r_{0}\right\}$ and the free boundary is the circle $\Gamma=\left\{x:|x|=r_{0}\right\}$ (cf. Figure 4.1 where $\Gamma$ is the circle in black and the shaded region is the discrete coincidence set at 
level 8). A simple calculation shows that the measure $\bar{\lambda}$ is given by

$$
\int_{\Omega} v d \bar{\lambda}=-\int_{\Gamma} v\left[4\left(C_{1} / r_{0}\right)+r_{0}\right] d s \quad \forall v \in C(\bar{\Omega})
$$

and hence

$$
\bar{\lambda}(\Omega)=-\left[4\left(C_{1} / r_{0}\right)+r_{0}\right]\left(2 \pi r_{0}\right) \approx 15.9838 .
$$

We compute $\bar{\lambda}_{h}(\Omega)=\sum_{p \in \mathcal{V}_{h}} c_{h, p}$ and report their values in Table 4.3, which agrees with Lemma 3.3 and Corollary 3.9.

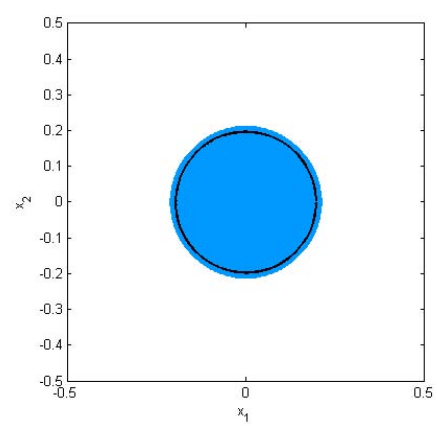

\begin{tabular}{|c|c|c|c|c|c|c|c|c|}
\hline Level & 1 & 2 & 3 & 4 & 5 & 6 & 7 & 8 \\
\hline $\bar{\lambda}_{j}(\Omega)$ & 13.064 & 18.026 & 16.287 & 16.076 & 16.004 & 15.995 & 15.986 & 15.984 \\
\hline
\end{tabular}

Figure 4.1: Discrete coincidence set at level 8 for Example 1

\section{Example 2}

We take $\Omega$ to be the unit square $(-0.5,0.5)^{2}, \psi(x)=0.1, y_{d}(x)=\sin \left(2 \pi\left(x_{1}+0.5\right)\left(x_{2}+0.5\right)\right)$, and $\beta=10^{-3}$. Since we do not know the exact solution, the errors are estimated from $\tilde{e}_{u, j}=\bar{u}_{j-1}-\bar{u}_{j}$. The $L_{2}$ and $H^{1}$ errors are reported in Table 4.4 and Table 4.5. We observe similar behavior as in Example 1. Since the free boundary is smooth (cf. Figure 4.2), the constraint function is a constant and all the angles of $\Omega$ are right angles, the solution $\bar{y}$ of (1.2) is a piecewise $H^{4}$ function that is globally $H^{3}$. The observed convergence for $\bar{u}_{h, 2}$ and $\bar{u}_{h, 3}$ in the $L_{2}$ and $H^{1}$ norms can therefore be explained as in Example 1.

In Table 4.6 we display $\left\|\bar{u}_{h, i}-\bar{u}_{h, j}\right\|_{L_{2}(\Omega)}$ for $1 \leq i<j<3$. The results indicate that the outputs of the three procedures are approximating the same optimal control and that $\bar{u}_{h, 2}$ and $\bar{u}_{h, 3}$ are closer to each other. We also report the values of $\bar{\lambda}_{h}(\Omega)$ in Table 4.7, which confirms Lemma 3.3.

\section{Example 3}

We take $\Omega$ to be the pentagonal domain obtained from the unit square $(-0.5,0.5)^{2}$ by removing the triangle with vertices $(0.5,0),(0.5,0.5)$ and $(0,0.5)$, and we use the same data as in Example 2 . The $L_{2}$ and $H^{1}$ errors are presented in Table 4.8 and Table 4.9 .

For this example, due to the two singular corners where the interior angle is larger than a right angle, the elliptic regularity index $\alpha$ can be any number $<1 / 3$. We observe that the orders of convergence in the $L_{2}$ norm for $\bar{u}_{h, 1}$ and $\bar{u}_{h, 2}$ are approaching $1 / 3$, but the errors for $\bar{u}_{h, 2}$ is smaller. On the other hand, the order of convergence in the $L_{2}$ norm for $\bar{u}_{h, 3}$ remains above 1 , and the magnitude of the error at level 8 is one order smaller than those from the other two procedures. 
Table 4.4: $L_{2}$ control errors for Example 2

\begin{tabular}{|c|cc|cc|cc|}
\hline & \multicolumn{2}{|c|}{ Procedure 1 } & \multicolumn{2}{c|}{ Procedure 2 } & \multicolumn{2}{c|}{ Procedure 3 } \\
\hline & \multicolumn{2}{|c|}{$\left\|\bar{u}_{8}\right\|_{L_{2}(\Omega)}=3.1450$} & \multicolumn{2}{|c|}{$\left\|\bar{u}_{8}\right\|_{L_{2}(\Omega)}=3.1456$} & \multicolumn{2}{|c|}{$\left\|\bar{u}_{8}\right\|_{L_{2}(\Omega)}=3.1464$} \\
\hline$j$ & $\frac{\left\|\tilde{e}_{u, j}\right\|_{L_{2}(\Omega)}}{\left\|\bar{u}_{8}\right\|_{L_{2}(\Omega)}}$ & order & $\frac{\left\|\tilde{e}_{u, j}\right\|_{L_{2}(\Omega)}}{\left\|\bar{u}_{8}\right\|_{L_{2}(\Omega)}}$ & order & $\frac{\left\|\tilde{e}_{u, j}\right\|_{L_{2}(\Omega)}}{\left\|\bar{u}_{\|}\right\|_{L_{2}(\Omega)}}$ & order \\
\hline 1 & $1.7360 \mathrm{E}+00$ & & $2.6748 \mathrm{E}+00$ & & $1.7293 \mathrm{E}+00$ & \\
2 & $1.0569 \mathrm{E}+00$ & 0.72 & $1.5626 \mathrm{E}+00$ & 0.78 & $1.5158 \mathrm{E}+00$ & 0.19 \\
3 & $6.7967 \mathrm{E}-01$ & 0.64 & $9.4478 \mathrm{E}-01$ & 0.73 & $7.5252 \mathrm{E}-01$ & 1.01 \\
4 & $3.5685 \mathrm{E}-01$ & 0.93 & $3.5294 \mathrm{E}-01$ & 1.42 & $2.1943 \mathrm{E}-01$ & 1.78 \\
5 & $1.7750 \mathrm{E}-01$ & 1.01 & $1.2171 \mathrm{E}-01$ & 1.54 & $7.1669 \mathrm{E}-02$ & 1.61 \\
6 & $8.5778 \mathrm{E}-02$ & 1.05 & $4.1983 \mathrm{E}-02$ & 1.54 & $2.8296 \mathrm{E}-02$ & 1.34 \\
7 & $4.1942 \mathrm{E}-02$ & 1.03 & $1.3128 \mathrm{E}-02$ & 1.68 & $8.1697 \mathrm{E}-03$ & 1.79 \\
8 & $2.0785 \mathrm{E}-02$ & 1.01 & $4.4419 \mathrm{E}-03$ & 1.56 & $2.9445 \mathrm{E}-03$ & 1.47 \\
\hline
\end{tabular}

Table 4.5: $H^{1}$ control errors for Example 2

\begin{tabular}{|c|ccc|ccc|}
\hline & \multicolumn{4}{|c|}{ Procedure 2} & \multicolumn{3}{c|}{ Procedure 3 } \\
\hline$j$ & $\left|\bar{u}_{j}\right|_{H^{1}(\Omega)}$ & $\frac{\left|\tilde{e}_{u, j}\right|_{H^{1}(\Omega)}}{\left|\bar{u}_{8}\right|_{H^{1}(\Omega)}}$ & order & $\left|\bar{u}_{j}\right|_{H^{1}(\Omega)}$ & $\frac{\left|\tilde{e}_{u, j}\right|_{H^{1}(\Omega)}}{\left|\bar{u}_{8}\right|_{H^{1}(\Omega)}}$ & order \\
\hline 1 & $7.4029 \mathrm{E}+01$ & $2.1478 \mathrm{E}+00$ & & $7.0996 \mathrm{E}+01$ & $1.7940 \mathrm{E}+00$ & \\
2 & $9.5938 \mathrm{E}+01$ & $2.3224 \mathrm{E}+00$ & -0.11 & $5.9649 \mathrm{E}+01$ & $1.5852 \mathrm{E}+00$ & 0.18 \\
3 & $7.8502 \mathrm{E}+01$ & $2.3951 \mathrm{E}+00$ & -0.04 & $4.6733 \mathrm{E}+01$ & $1.4272 \mathrm{E}+00$ & 0.15 \\
4 & $6.2256 \mathrm{E}+01$ & $1.8227 \mathrm{E}+00$ & 0.39 & $4.1926 \mathrm{E}+01$ & $8.6035 \mathrm{E}-01$ & 0.73 \\
5 & $5.0004 \mathrm{E}+01$ & $1.2653 \mathrm{E}+00$ & 0.53 & $3.9514 \mathrm{E}+01$ & $5.2965 \mathrm{E}-01$ & 0.70 \\
6 & $4.3651 \mathrm{E}+01$ & $8.5364 \mathrm{E}-01$ & 0.57 & $3.8649 \mathrm{E}+01$ & $3.8452 \mathrm{E}-01$ & 0.46 \\
7 & $4.0475 \mathrm{E}+01$ & $5.4839 \mathrm{E}-01$ & 0.64 & $3.8146 \mathrm{E}+01$ & $2.1913 \mathrm{E}-01$ & 0.81 \\
8 & $3.9078 \mathrm{E}+01$ & $3.6370 \mathrm{E}-01$ & 0.59 & $3.7977 \mathrm{E}+01$ & $1.5217 \mathrm{E}-01$ & 0.53 \\
\hline
\end{tabular}

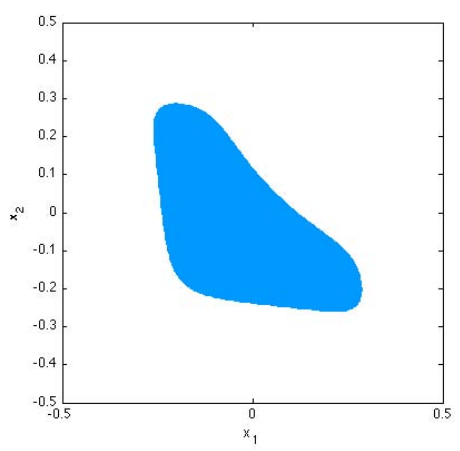

Figure 4.2: Discrete coincidence set at level 8 for Example 2

The numerical results in Table 4.9 shows that $\left|\bar{u}_{h, 2}\right|_{H^{1}(\Omega)}$ is growing and $\bar{u}_{h, 2}$ does not converge in $H^{1}(\Omega)$. However $\left|\bar{u}_{h, 3}\right|_{H^{1}(\Omega)}$ remains bounded and the order of convergence in the $H^{1}$ norm for $\bar{u}_{h, 3}$ appears to be $1 / 2$.

The values of $\bar{\lambda}_{h}(\Omega)$ are presented in Table 4.10, which confirms Lemma 3.3.

We also display the plots of the discrete optimal controls from the three procedures in Figure 4.3. The discrete controls $\bar{u}_{h, 1}$ from the first post-processing procedure exhibits strong singular behavior near the two singular corners (which is the reason that we only show the plot for level 6). The discrete control $\bar{u}_{h, 2}$ from the second procedure, 
Table 4.6: Comparison of $\bar{u}_{h, 1}, \bar{u}_{h, 2}$ and $\bar{u}_{h, 3}$ for Example 2

\begin{tabular}{|c|c|c|c|}
\hline Level & $\left\|\bar{u}_{h, 1}-\bar{u}_{h, 2}\right\|_{L_{2}(\Omega)}$ & $\left\|\bar{u}_{h, 1}-\bar{u}_{h, 3}\right\|_{L_{2}(\Omega)}$ & $\left\|\bar{u}_{h, 2}-\bar{u}_{h, 3}\right\|_{L_{2}(\Omega)}$ \\
\hline 1 & $5.7595 \mathrm{E}+00$ & $5.4684 \mathrm{E}+00$ & $1.6669 \mathrm{E}+00$ \\
2 & $3.4365 \mathrm{E}+00$ & $3.0844 \mathrm{E}+00$ & $1.6997 \mathrm{E}+00$ \\
3 & $1.4932 \mathrm{E}+00$ & $1.5337 \mathrm{E}+00$ & $8.4103 \mathrm{E}-01$ \\
4 & $6.7293 \mathrm{E}-01$ & $7.1295 \mathrm{E}-01$ & $3.2850 \mathrm{E}-01$ \\
5 & $3.1200 \mathrm{E}-01$ & $3.2310 \mathrm{E}-01$ & $1.0996 \mathrm{E}-01$ \\
6 & $1.5200 \mathrm{E}-01$ & $1.5444 \mathrm{E}-01$ & $3.6053 \mathrm{E}-02$ \\
7 & $7.5158 \mathrm{E}-02$ & $7.5592 \mathrm{E}-02$ & $1.1728 \mathrm{E}-02$ \\
8 & $3.7490 \mathrm{E}-02$ & $3.7576 \mathrm{E}-02$ & $3.9451 \mathrm{E}-03$ \\
\hline
\end{tabular}

Table 4.7: $\bar{\lambda}_{h}(\Omega)$ for Example 2

\begin{tabular}{|l|c|c|c|c|c|c|c|c|}
\hline Level & 1 & 2 & 3 & 4 & 5 & 6 & 7 & 8 \\
\hline $\bar{\lambda}_{j}(\Omega)$ & 0.13180 & 0.25789 & 0.25950 & 0.25953 & 0.26036 & 0.26063 & 0.26072 & 0.26075 \\
\hline
\end{tabular}

Table 4.8: $L_{2}$ control errors for Example 3

\begin{tabular}{|c|cc|cc|cc|}
\hline & \multicolumn{2}{|c|}{ Procedure 1 } & \multicolumn{2}{c|}{ Procedure 2 } & \multicolumn{2}{c|}{ Procedure 3 } \\
\hline & \multicolumn{2}{|c|}{$\left\|\bar{u}_{8}\right\|_{L_{2}(\Omega)}=2.7744$} & \multicolumn{2}{|c|}{$\left\|\bar{u}_{8}\right\|_{L_{2}(\Omega)}=2.7729$} & \multicolumn{2}{|c|}{$\left\|\bar{u}_{8}\right\|_{L_{2}(\Omega)}=2.7759$} \\
\hline$j$ & $\frac{\left\|\tilde{e}_{u, j}\right\|_{L_{2}(\Omega)}}{\left\|\tilde{u}_{8}\right\|_{L_{2}(\Omega)}}$ & order & $\frac{\left\|\tilde{e}_{u, j}\right\|_{L_{2}(\Omega)}}{\left\|\bar{u}_{8}\right\|_{L_{2}(\Omega)}}$ & order & $\frac{\left\|\tilde{e}_{u, j}\right\|_{L_{2}(\Omega)}}{\left\|\bar{u}_{8}\right\|_{L_{2}(\Omega)}}$ & order \\
\hline 1 & $2.2444 \mathrm{E}+00$ & & $1.7291 \mathrm{E}+00$ & & $1.7162 \mathrm{E}+00$ & \\
2 & $1.5563 \mathrm{E}+00$ & 0.53 & $1.0841 \mathrm{E}+00$ & 0.67 & $8.5845 \mathrm{E}-01$ & 1.00 \\
3 & $7.6236 \mathrm{E}-01$ & 1.03 & $3.9368 \mathrm{E}-01$ & 1.46 & $2.4613 \mathrm{E}-01$ & 1.80 \\
4 & $3.9444 \mathrm{E}-01$ & 0.95 & $1.3651 \mathrm{E}-01$ & 1.53 & $8.0146 \mathrm{E}-02$ & 1.62 \\
5 & $2.2574 \mathrm{E}-01$ & 0.81 & $5.3314 \mathrm{E}-02$ & 1.36 & $3.2336 \mathrm{E}-02$ & 1.31 \\
6 & $1.4719 \mathrm{E}-01$ & 0.62 & $2.5368 \mathrm{E}-02$ & 1.07 & $9.6051 \mathrm{E}-03$ & 1.75 \\
7 & $1.0606 \mathrm{E}-01$ & 0.47 & $1.7158 \mathrm{E}-02$ & 0.56 & $3.6245 \mathrm{E}-03$ & 1.41 \\
8 & $8.0644 \mathrm{E}-02$ & 0.40 & $1.3122 \mathrm{E}-02$ & 0.39 & $1.4156 \mathrm{E}-03$ & 1.36 \\
\hline
\end{tabular}

Table 4.9: $H^{1}$ control errors for Example 3

\begin{tabular}{|c|ccc|ccc|}
\hline & \multicolumn{7}{|c|}{ Procedure 2 } & \multicolumn{3}{c|}{ Procedure 3 } \\
\hline$j$ & $\left|\bar{u}_{j}\right|_{H^{1}(\Omega)}$ & $\frac{\left|\tilde{e}_{u, j}\right|_{H^{1}(\Omega)}}{\left|\bar{u}_{8}\right|_{H^{1}(\Omega)}}$ & order & $\left|\bar{u}_{j}\right|_{H^{1}(\Omega)}$ & $\frac{\left|\tilde{e}_{u, j}\right|_{H^{1}(\Omega)}}{\left|\bar{u}_{8}\right|_{H^{1}(\Omega)}}$ & order \\
\hline 1 & $9.4377 \mathrm{E}+01$ & $1.0738 \mathrm{E}+00$ & & $5.7162 \mathrm{E}+01$ & $1.8091 \mathrm{E}+00$ & \\
2 & $7.7411 \mathrm{E}+01$ & $1.1227 \mathrm{E}+00$ & -0.06 & $4.3027 \mathrm{E}+01$ & $1.6849 \mathrm{E}+00$ & 0.10 \\
3 & $5.9334 \mathrm{E}+01$ & $8.4377 \mathrm{E}-01$ & 0.41 & $3.7305 \mathrm{E}+01$ & $9.9719 \mathrm{E}-01$ & 0.76 \\
4 & $4.7355 \mathrm{E}+01$ & $5.9996 \mathrm{E}-01$ & 0.49 & $3.4503 \mathrm{E}+01$ & $6.1036 \mathrm{E}-01$ & 0.71 \\
5 & $4.3569 \mathrm{E}+01$ & $4.7282 \mathrm{E}-01$ & 0.34 & $3.3552 \mathrm{E}+01$ & $4.4983 \mathrm{E}-01$ & 0.44 \\
6 & $4.6932 \mathrm{E}+01$ & $4.8583 \mathrm{E}-01$ & -0.04 & $3.2965 \mathrm{E}+01$ & $2.5828 \mathrm{E}-01$ & 0.80 \\
7 & $5.9210 \mathrm{E}+01$ & $6.7824 \mathrm{E}-01$ & -0.48 & $3.2737 \mathrm{E}+01$ & $1.7873 \mathrm{E}-01$ & 0.53 \\
8 & $8.3754 \mathrm{E}+01$ & $1.0472 \mathrm{E}+00$ & -0.63 & $3.2657 \mathrm{E}+01$ & $1.2544 \mathrm{E}-01$ & 0.51 \\
\hline
\end{tabular}

which has an averaging effect, is not as singular near the two singular corners. The discrete control $\bar{u}_{h, 3}$ from the third procedure, which has a smoothing effect, does not exhibit any singular behavior. 
Table 4.10: $\bar{\lambda}_{h}(\Omega)$ for Example 3

\begin{tabular}{|c|c|c|c|c|c|c|c|c|}
\hline Level & 1 & 2 & 3 & 4 & 5 & 6 & 7 & 8 \\
\hline $\bar{\lambda}_{j}(\Omega)$ & 0.26516 & 0.26509 & 0.26525 & 0.26618 & 0.26655 & 0.26672 & 0.26680 & 0.26684 \\
\hline
\end{tabular}

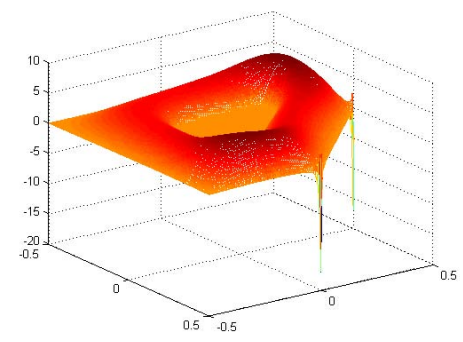

(a) $\bar{u}_{6}$ (Scheme 1)

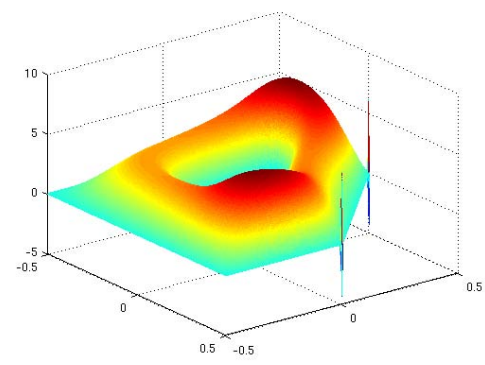

(b) $\bar{u}_{8}$ (Scheme 2)

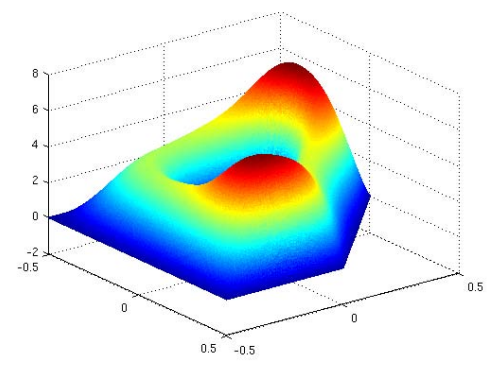

(c) $\bar{u}_{8}$ (Scheme 3)

Figure 4.3: The discrete controls for Example 3

\section{Example 4}

We solve the same problem in Example 3 on graded meshes, which are obtained from a uniform triangulation $\mathcal{T}_{0}$ by the refinement procedure from [5] (cf. Figure 4.4).
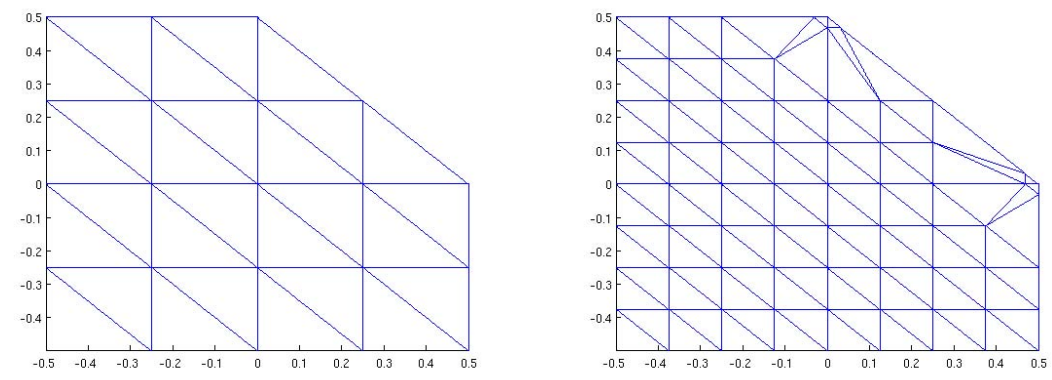

Figure 4.4: Triangulation $\mathcal{T}_{0}$ (left) and $\mathcal{T}_{1}$ (right) for the pentagonal domain

The $L_{2}$ and $H^{1}$ errors are presented in Table 4.11 and Table 4.12. We observe that the order of convergence in the $L_{2}$ norm for $\bar{u}_{h, 1}$ and $\bar{u}_{h, 2}$ are restored to 1 , and the magnitudes of the errors for $\bar{u}_{h, 2}$ are smaller. The order of convergence in the $L_{2}$ norm for $\bar{u}_{h, 3}$ is 1.5 , and the magnitude of the error at level 7 is one order smaller than those from the other two post-processing procedures. Similar to Example 3, $\left|\bar{u}_{h, 2}\right|_{H^{1}(\Omega)}$ is growing and $\bar{u}_{h, 2}$ does not converge in $H^{1}(\Omega)$. But $\left|\bar{u}_{h, 3}\right|_{H^{1}(\Omega)}$ remains bounded and the order of convergence in the $H^{1}$ norm for $\bar{u}_{h, 3}$ again appears to be $1 / 2$.

The values of $\bar{\lambda}_{h}(\Omega)$ are presented in Table 4.13, which confirms Lemma 3.3 and also agrees with the results in Table 4.10. The discrete coincidence set at level 8 for Example 3 and the discrete coincidence set at level 7 for Example 4 are displayed in Figure 4.5. They appear to be identical.

Finally we display the plots for the discrete optimal controls from the three procedures in Figure 4.6. We observe even stronger singular behaviors of $\bar{u}_{h, 1}$ and $\bar{u}_{h, 2}$ near the singular corners, since the triangles near these corners are substantially smaller in graded meshes, but the averaging effect of the Procedure 2 still renders the singular behavior of $\bar{u}_{h, 2}$ less pronounced than that of $\bar{u}_{h, 1}$. On the other hand, $\bar{u}_{h, 3}$ does not exhibit any singular behavior because of the smoothing effect of Procedure 3. The plot of $\bar{u}_{h, 3}$ at level 8 in Figure 4.3 and the plot of $\bar{u}_{h, 3}$ at level 7 in Figure 4.6 are 
Table 4.11: $L_{2}$ control errors for Example 4

\begin{tabular}{|c|cc|cc|cr|}
\hline & \multicolumn{2}{|c|}{ Procedure 1 } & \multicolumn{2}{c|}{ Procedure 2 } & \multicolumn{2}{c|}{ Procedure 3 } \\
\hline & $\left\|\bar{u}_{7}\right\|_{L_{2}(\Omega)}=2.7733$ & $\left\|\bar{u}_{7}\right\|_{L_{2}(\Omega)}=2.7732$ & $\left\|\bar{u}_{7}\right\|_{L_{2}(\Omega)}=2.7747$ \\
\hline$j$ & $\frac{\left\|\tilde{e}_{u, j}\right\|_{L_{2}(\Omega)}}{\left\|\bar{u}_{7}\right\|_{L_{2}(\Omega)}}$ & order & $\frac{\left\|\tilde{e}_{u, j}\right\|_{L_{2}(\Omega)}}{\left\|\bar{u}_{7}\right\|_{L_{2}(\Omega)}}$ & order & $\frac{\left\|\tilde{e}_{u, j}\right\|_{L_{2}(\Omega)}}{\left\|\bar{u}_{7}\right\|_{L_{2}(\Omega)}}$ & order \\
\hline 1 & $5.5682 \mathrm{E}-01$ & & $4.7166 \mathrm{E}-01$ & & $1.5870 \mathrm{E}+00$ & \\
2 & $3.7755 \mathrm{E}-01$ & 0.56 & $2.9528 \mathrm{E}-01$ & 0.68 & $5.0011 \mathrm{E}-01$ & 1.67 \\
3 & $2.1478 \mathrm{E}-01$ & 0.81 & $1.5324 \mathrm{E}-01$ & 0.95 & $1.4282 \mathrm{E}-01$ & 1.81 \\
4 & $1.1507 \mathrm{E}-01$ & 0.90 & $7.7635 \mathrm{E}-02$ & 0.98 & $5.4091 \mathrm{E}-02$ & 1.40 \\
5 & $6.2214 \mathrm{E}-02$ & 0.89 & $4.0842 \mathrm{E}-02$ & 0.93 & $1.5540 \mathrm{E}-02$ & 1.80 \\
6 & $3.3545 \mathrm{E}-02$ & 0.89 & $2.1646 \mathrm{E}-02$ & 0.92 & $5.6753 \mathrm{E}-03$ & 1.45 \\
7 & $1.7948 \mathrm{E}-02$ & 0.90 & $1.1353 \mathrm{E}-02$ & 0.93 & $1.9061 \mathrm{E}-03$ & 1.57 \\
\hline
\end{tabular}

Table 4.12: $H^{1}$ control errors for Example 4

\begin{tabular}{|c|ccc|ccc|}
\hline & \multicolumn{3}{|c|}{ Procedure 2 } & \multicolumn{3}{c|}{ Procedure 3 } \\
\hline$j$ & $\left|\bar{u}_{j}\right|_{H^{1}(\Omega)}$ & $\frac{\left|\tilde{e}_{u, j}\right|_{H^{1}(\Omega)}}{\left|\bar{u}_{7}\right|_{H^{1}(\Omega)}}$ & order & $\left|\bar{u}_{j}\right|_{H^{1}(\Omega)}$ & $\frac{\left|\tilde{e}_{u, j}\right|_{H^{1}(\Omega)}}{\left|\bar{u}_{7}\right|_{H^{1}(\Omega)}}$ & order \\
\hline 1 & $4.8945 \mathrm{E}+01$ & $4.1644 \mathrm{E}-04$ & & $5.3771 \mathrm{E}+01$ & $2.6689 \mathrm{E}+00$ & \\
2 & $1.4282 \mathrm{E}+02$ & $1.1541 \mathrm{E}-03$ & -1.47 & $4.1244 \mathrm{E}+01$ & $1.5051 \mathrm{E}+00$ & 0.83 \\
3 & $5.0717 \mathrm{E}+02$ & $4.1234 \mathrm{E}-03$ & -1.84 & $3.6627 \mathrm{E}+01$ & $8.0510 \mathrm{E}-01$ & 0.90 \\
4 & $1.9470 \mathrm{E}+03$ & $1.5822 \mathrm{E}-02$ & -1.94 & $3.3989 \mathrm{E}+01$ & $5.3368 \mathrm{E}-01$ & 0.59 \\
5 & $7.6660 \mathrm{E}+03$ & $6.2274 \mathrm{E}-02$ & -1.98 & $3.3170 \mathrm{E}+01$ & $2.8460 \mathrm{E}-01$ & 0.91 \\
6 & $3.0479 \mathrm{E}+04$ & $2.4755 \mathrm{E}-01$ & -1.99 & $3.2816 \mathrm{E}+01$ & $2.0225 \mathrm{E}-01$ & 0.49 \\
7 & $1.2167 \mathrm{E}+05$ & $9.8807 \mathrm{E}-01$ & -2.00 & $3.2691 \mathrm{E}+01$ & $1.3918 \mathrm{E}-01$ & 0.54 \\
\hline
\end{tabular}

Table 4.13: $\bar{\lambda}_{h}(\Omega)$ for Example 4

\begin{tabular}{|l|c|c|c|c|c|c|c|}
\hline Level & 1 & 2 & 3 & 4 & 5 & 6 & 7 \\
\hline $\bar{\lambda}_{j}(\Omega)$ & 0.24840 & 0.25973 & 0.26422 & 0.26605 & 0.26662 & 0.26681 & 0.26687 \\
\hline
\end{tabular}
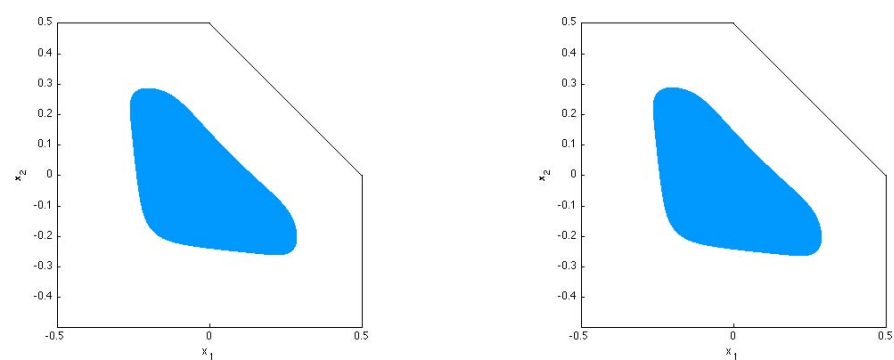

Figure 4.5: The discrete coincidence set at level 8 for Example 3 (left) and the discrete coincidence set at level 7 for Example 4 (right)

identical, and they are also identical to the plot of the optimal control in [6] generated by a partition of unity method for the same problem. 


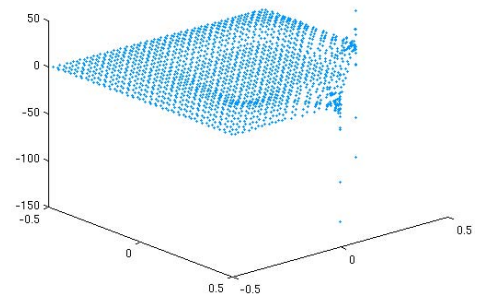

(a) $\bar{u}_{3}$ (Procedure 1)

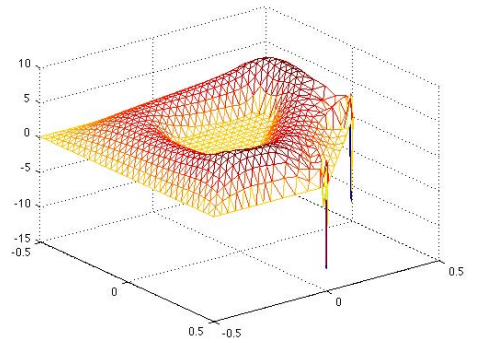

(b) $\bar{u}_{3}$ (Procedure 2)

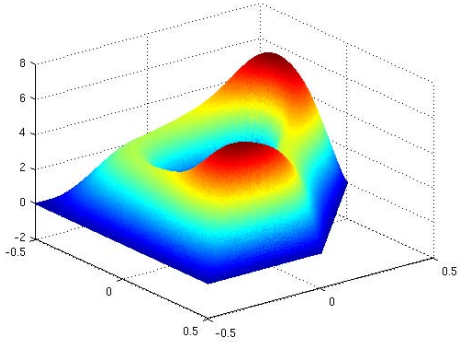

(c) $\bar{u}_{7}$ (Procedure 3)

Figure 4.6: The discrete controls for Example 4

\section{Concluding Remarks}

We consider three post-processing procedures that compute approximations of the optimal control from the approximation of the optimal state obtained by a quadratic $C^{0}$ interior penalty method. We have similar $L_{2}$ error estimates for the three procedures, but numerical results indicate that the procedure based on the solution of a Poisson problem outperforms the other two.

Finite element methods for state constrained elliptic distributed optimal control problems were developed in [19, 34], where the finite element approximation $\left(\bar{y}_{h}, \bar{u}_{h}\right)$ of $(\bar{y}, \bar{u})$ was obtained from discrete versions of the optimal control problems. In this approach the estimates for $\left\|\bar{y}-\bar{y}_{h}\right\|_{H^{1}(\Omega)}$ and $\left\|\bar{u}-\bar{u}_{h}\right\|_{L_{2}(\Omega)}$ are coupled and consequently have the same magnitude, which is $O\left(h^{1-\epsilon}\right)$ if $\Omega$ is smooth or a rectangle.

In contrast, the error estimate in [13] for the approximate optimal state computed by the quadratic $C^{0}$ interior penalty method is $O(h)$ for a $H^{2}$-like energy norm on general convex domains, provided that we use graded meshes around any corner where the interior angle is larger than a right angle. Since the $H^{1}$ norm is a weaker norm, the error in the $H^{1}$ norm is expected to be smaller. In practice we observe that on a rectangular domain the error for the state in the $H^{1}$ norm is $O\left(h^{2}\right)$, and on the pentagonal domain (cf. Example 3) it is $O(h)$ on uniform mesh and $O\left(h^{1.5}\right)$ on graded meshes.

We have also shown that on graded meshes the $L_{2}$ errors for the approximations of the optimal control obtained by post-processing are also $O(h)$ for Procedure 1 and Procedure 2, and $O\left(h^{1-\epsilon}\right)$ for Procedure 3. In practice we observe that on a rectangular domain the $L_{2}$ error for the control (Procedure 2 and Procedure 3 ) are $O\left(h^{1.5}\right)$, and on the pentagonal domain it is better than $O(h)$ for Procedure 3 even on quasi-uniform meshes.

Furthermore we observe that the $H^{1}$ error for the control obtained by Procedure 3 is $O\left(h^{0.5}\right)$ on both the rectangular domain and the pentagonal domain, for quasi-uniform meshes. But the theoretical justification of $H^{1}$ convergence remains open. Progress in this direction will also shed new light on the convergence of the discrete Lagrange multipliers $\bar{\lambda}_{h}$ (cf. Section 3.5).

\section{References}

[1] R. Adams, J. Fournier, Sobolev Spaces (Second Edition), Academic Press, Amsterdam, 2003.

[2] T. Apel, A.M. Sändig, J. Whiteman, Graded mesh refinement and error estimates for finite element solutions of elliptic boundary value problems in non-smooth domains, Math. Methods Appl. Sci. 19 (1996) 63-85.

[3] M. Bergounioux, K. Ito, K. Kunisch, Primal-dual strategy for constrained optimal control problems, SIAM J. Control Optim. 37 (1999) 1176-1194 (electronic).

[4] H. Blum, R. Rannacher, On the boundary value problem of the biharmonic operator on domains with angular corners, Math. Methods Appl. Sci. 2 (1980) 556-581.

[5] J. Brannick, H. Li, L. Zikatanov, Uniform convergence of the multigrid $V$-cycle on graded meshes for corner singularities, Numer. Linear Algebra Appl. 15 (2008) 291-306.

[6] S. Brenner, C. Davis, L.Y. Sung, A partition of unity method for a class of fourth order elliptic variational inequalities, Comp. Methods Appl. Mech. Engrg. (2014) 612-626.

[7] S.C. Brenner, C. Carstensen, Finite Element Methods, in: E. Stein, R. de Borst, T. Hughes (Eds.), Encyclopedia of Computational Mechanics, Wiley, Weinheim, 2004, pp. 73-118. 
[8] S.C. Brenner, M. Neilan, L.Y. Sung, Isoparametric $C^{0}$ interior penalty methods for plate bending problems on smooth domains, Calcolo 49 (2013) 35-67.

[9] S.C. Brenner, L.R. Scott, The Mathematical Theory of Finite Element Methods (Third Edition), Springer-Verlag, New York, 2008.

[10] S.C. Brenner, L.Y. Sung, $C^{0}$ interior penalty methods for fourth order elliptic boundary value problems on polygonal domains, J. Sci. Comput. 22/23 (2005) 83-118.

[11] S.C. Brenner, L.Y. Sung, H. Zhang, Y. Zhang, A quadratic $C^{0}$ interior penalty method for the displacement obstacle problem of clamped Kirchhoff plates, SIAM J. Numer. Anal. 50 (2012) 3329-3350.

[12] S.C. Brenner, L.Y. Sung, Y. Zhang, Finite element methods for the displacement obstacle problem of clamped plates, Math. Comp. 81 (2012) 1247-1262.

[13] S.C. Brenner, L.Y. Sung, Y. Zhang, A quadratic $C^{0}$ interior penalty method for an elliptic optimal control problem with state constraints, in: O.K. X. Feng, Y. Xing (Eds.), Recent Developments in Discontinuous Galerkin Finite Element Methods for Partial Differential Equations, volume 157 of The IMA Volumes in Mathematics and its Applications, Springer, Cham-Heidelberg-New York-Dordrecht-London, 2013, pp. 97-132. (2012 John H. Barrett Memorial Lectures).

[14] L. Caffarelli, A. Friedman, The obstacle problem for the biharmonic operator, Ann. Scuola Norm. Sup. Pisa Cl. Sci. (4) 6 (1979) $151-184$.

[15] E. Casas, Control of an elliptic problem with pointwise state constraints, SIAM J. Control Optim. 24 (1986) $1309-1318$.

[16] S. Cherednichenko, A. Rösch, Error estimates for the discretization of elliptic control problems with pointwise control and state constraints, Comput. Optim. Appl. 44 (2009) 27-55.

[17] P. Ciarlet, The Finite Element Method for Elliptic Problems, North-Holland, Amsterdam, 1978.

[18] M. Dauge, Elliptic Boundary Value Problems on Corner Domains, Lecture Notes in Mathematics 1341, Springer-Verlag, Berlin-Heidelberg, 1988.

[19] K. Deckelnick, M. Hinze, Convergence of a finite element approximation to a state-constrained elliptic control problem, SIAM J. Numer. Anal. 45 (2007) 1937-1953 (electronic).

[20] G. Engel, K. Garikipati, T.J.R. Hughes, M.G. Larson, L. Mazzei, R.L. Taylor, Continuous/discontinuous finite element approximations of fourth order elliptic problems in structural and continuum mechanics with applications to thin beams and plates, and strain gradient elasticity, Comput. Methods Appl. Mech. Engrg. 191 (2002) 3669-3750.

[21] J. Frehse, Zum Differenzierbarkeitsproblem bei Variationsungleichungen höherer Ordnung, Abh. Math. Sem. Univ. Hamburg 36 (1971) $140-149$.

[22] J. Frehse, On the regularity of the solution of the biharmonic variational inequality, Manuscripta Math. 9 (1973) 91-103.

[23] A. Friedman, Variational Principles and Free-Boundary Problems (Second Edition), Robert E. Krieger Publishing Co. Inc., Malabar, FL, 1988.

[24] W. Gong, N. Yan, A mixed finite element scheme for optimal control problems with pointwise state constraints, J. Sci. Comput. 46 (2011) $182-203$.

[25] P. Grisvard, Elliptic Problems in Non Smooth Domains, Pitman, Boston, 1985.

[26] P. Grisvard, Singularities in Boundary Value Problems, Masson, Paris, 1992.

[27] M. Hintermüller, K. Ito, K. Kunisch, The primal-dual active set strategy as a semismooth Newton method, SIAM J. Optim. 13 (2003) $865-888$.

[28] M. Hintermüller, K. Kunisch, PDE-constrained optimization subject to pointwise constraints on the control, the state, and its derivative, SIAM J. Optim. 20 (2009) 1133-1156.

[29] D. Kinderlehrer, G. Stampacchia, An Introduction to Variational Inequalities and Their Applications, Society for Industrial and Applied Mathematics, Philadelphia, 2000.

[30] K. Krumbiegel, C. Meyer, A. Rösch, A priori error analysis for linear quadratic elliptic Neumann boundary control problems with control and state constraints, SIAM J. Control Optim. 48 (2010) 5108-5142.

[31] P. Lax, Functional Analysis, Wiley-Interscience, New York, 2002.

[32] J.L. Lions, G. Stampacchia, Variational inequalities, Comm. Pure Appl. Math. 20 (1967) 493-519.

[33] W. Liu, W. Gong, N. Yan, A new finite element approximation of a state-constrained optimal control problem, J. Comput. Math. 27 (2009) 97-114.

[34] C. Meyer, Error estimates for the finite-element approximation of an elliptic control problem with pointwise state and control constraints, Control Cybernet. 37 (2008) 51-83.

[35] S. Nazarov, B. Plamenevsky, Elliptic Problems in Domains with Piecewise Smooth Boundaries, de Gruyter, Berlin-New York, 1994.

[36] I. Neitzel, U. Prüfert, T. Slawig, A smooth regularization of the projection formula for constrained parabolic optimal control problems, Numer. Funct. Anal. Optim. 32 (2011) 1283-1315.

[37] J. Nečas, Les Méthodes Directes en Théorie des Équations Elliptiques, Masson, Paris, 1967.

[38] J.F. Rodrigues, Obstacle Problems in Mathematical Physics, North-Holland Publishing Co., Amsterdam, 1987.

[39] A. Rösch, S. Steinig, A priori error estimates for a state-constrained elliptic optimal control problem, ESAIM Math. Model. Numer. Anal. 46 (2012) 1107-1120. 
impression that the classical approaches of [17, 28] or [CR09] are more flexible. The authors should comment on this in the introduction.

This point is well taken. We have certainly not worked out all the optimal control problems that can be solved efficiently as fourth order variational inequalities.

Here is what we know.

- Our method and analysis can be extended to optimal control problems of the form

$$
\begin{aligned}
\text { minimize } & J(y, u)=\frac{1}{2} \int_{\Omega}\left(y-y_{d}\right)^{2} d x+\frac{\beta}{2} \int_{\Omega}\left(u-u_{d}\right)^{2} d x \\
\text { over } & (y, u) \in H_{0}^{1}(\Omega) \times L_{2}(\Omega) \\
\text { subject to } & \left\{\begin{aligned}
-\Delta y+\gamma y=u & \text { in } \Omega \\
y \leq \psi & \text { a.e. in } \Omega
\end{aligned}\right.
\end{aligned}
$$

where $\gamma \geq 0$ and $u_{d} \in H_{0}^{1}(\Omega)$. The Laplace operator can also be replaced by a second order uniformly elliptic operator with sufficiently smooth coefficients. We can handle similar problems with Neumann boundary conditions. We have also found efficient adaptive algorithms for these optimal control problems that are based on the $C^{0}$ interior penalty approach. These are all work in progress.

- It may also be possible to solve problems with both state and control constraints by our approach, since the regularity of biharmonic variational inequalities with pointwise constraints on the Laplacian was obtained by Brézis and Stampacchia in 1977.

- The assumption on the upper bound $\psi$ can be relaxed to $\psi \epsilon$ $W^{2, \infty}(\Omega)$, which is also the assumption that appears in DeckelnickHinze (2007) and Meyer (2008).

Since the focus of our paper is on a particular postprocessing procedure and much of our work on optimal control problems are still in progress, we do not think it is appropriate to include a lengthy comparison of the merits of the different approaches in this paper. Therefore we only mention in Remark 1.1 that it would be interesting to find out whether 
our approach can be extended to problems with both state and control constraints and also point to some references for such problems.

4. I am surprised about the limited rate of convergence in the case of a convex polygon with largest angle more than $\pi / 2$. An inspection of the error estimates in $[17,28]$ shows that these roughly correspond to the square root of the $L^{\infty}$-error of the state equation. Thus, if $\Omega$ is just a convex polygon, then the $H^{2}$-regularity yields an $L^{\infty}$-convergence rate of 1 in two dimensions giving in turn an order of convergence of $1 / 2-\varepsilon$, $\varepsilon>0$, for the optimal control. In comparison, the authors' result does not seem to yield any rate of convergence if the largest angle tends to $\pi$ (as $\alpha=0$ in this case). Therefore, I also expect their mesh grading to be not optimal. Why is the convergence rate so bad, if the largest angle is greater than $\pi / 2$ ?

This is due to the difference between the regularity results for second order and fourth order problems on nonsmooth domains. Since we are solving a fourth order problem, the conditions for regularity are more stringent. The trade-off is that we have more information on the behavior of the optimal state since we have convergence in an $H^{2}$-like energy norm. The singular behavior as the largest angle approaches $\pi$ can be overcome by graded meshes as demonstrated in this paper, or by including the known singular functions into the approximation space as demonstrated in Brenner-Davis-Sung (2014).

\section{$\underline{\text { Reviewer } 2}$}

1. Please clarify what do you mean by "provide an approximation .... on" in the Remark 2.2.

We mean that the boundary value problem of simply supported plates can be solved by using the bilinear form $a_{h}(\cdot, \cdot)$. We have rewritten Remark 2.2 to clarify this.

2. It is affirm that bilinear form defined in (2.5) is positive definite, how depend this on the penalty parameters?. In fact, in the numerical examples these parameter are fixed without any comments.

The bilinear form in (2.4) (and hence the bilinear form in (2.5)) is positive definite if the penalty parameter is sufficiently large. The choice 
of $\sigma$ depends on the shape regularity of the triangulation. This is now mentioned after (2.4).

Based on our experience, $\sigma=5$ is sufficient for uniform meshes. For graded meshes, which are less regular, we have to use a larger $\sigma$. We have now pointed this out at the beginning of Section 4.

3. Because the analyzed scheme is based on the solution of a Poisson problem, this procedure seems to be better, however the computational requirement should be highest. This is mentioned in the paper, but a measure of the difference should be included (for instance, cpu time)?.

We have not compared the costs of the three procedures since the computational cost is dominated by the cost of solving the variational inequality. We have now mentioned this at the beginning of Section 4 .

We have also pointed to the paper by Bergounioux, Ito and Kunisch in the new Remark 1.1. 